\title{
On the integrability problem for systems of partial differential equations in one unknown function, II
}

\author{
Antonio Kumpera
}

\begin{abstract}
We continue here our discussion of Part I, [18], by examining the local equivalence problem for partial differential equations and illustrating it with some examples, since almost any integration process or method is actually a local equivalence problem involving a suitable model. We terminate the discussion by inquiring on non-integrable Pfaffian systems and on their integral manifolds of maximal dimension.
\end{abstract}

Анотація. В статті продовжується обговорення проблеми локальної еквівалентності (фактично інтегровності) неінтегровних систем Пфаффа та їх інтегральних підноговидів $\mathcal{S}$ максимальних розмірностей, розпочате в частині I, [18].

Спочатку ми нагадуємо означення різних типів дужок та будуємо характеристичні векторні поля для контактних систем Пфаффа $\mathcal{C}(\mathcal{S})$ асоційованих з $\mathcal{S}$ та показуємо, що інтегровність системи еквівалентна такій аналітичній властивості: дужка Лагранжа будь-яких двох функцій, що дорівнюють нулю на $\mathcal{S}$ також зануляється на $\mathcal{S}$. Це дозволяє працювати з локально з системою рівнянь $\left\{f_{\alpha}=0\right\}$, що задають систему $\mathcal{S}$.

Далі розглядаються інтегральні контактні елементи вищого порядку в контексті грассманових розшарувань та досліджуємо як інтегральні елементи певної розмірності та заданого порядку можуть бути розширені до інтегральних контактних елементів на одиницю вищої розмірності. Також обговорюється локальна еквівалентність різних типів рівнянь та локальна еквівалентніть групоїдів Лі.

В кінці роботи обговорюються неінтегровні системи Пфаффа та їх обмеження на інтегральні підмноговиди максимальних розмірностей. Ми також описуємо конструкцію поняття характера системи Пфаффа введене Е. Картаном, та похідної системи, яка має менший ранг, а послідність похідних систем дозволяе знаходити максимальні інтегральні підмноговиди початкової системи.

2010 Mathematics Subject Classification: Primary 53C05; Secondary 53C15, 53C17

Keywords: Partial differential equations, Pfaffian system, contact structures, local equivalence. 


\section{CONTENTS}

\section{Part II}

1 Introduction

2 Brackets

3 Higher order differential equations

4 The local equivalence problem

5 The local equivalence of differential systems

6 The local equivalence of Lie groupoids

7 Integral contact elements

8 Integral sub-manifolds

9 The derived systems

10 The pseudo-groups of local automorphisms

11 Pfaffian systems whose characters are equal to one

12 The Cartan co-variant approach

13 Integration of Pfaffian systems with character equal to one

14 Pfaffian systems whose characters are larger than one

15 Singular Pfaffian Systems

16 Examples

\section{INTRODUCTION}

We begin by defining several brackets and construct characteristic vector fields of $\mathcal{C}(\mathcal{S})$, the contact Pfaffian system associated to $\mathcal{S}$, via the geometry of contact transformations, proving subsequently that integrability is equivalent to the following analytical condition: The Lagrangean bracket of any two functions vanishing on $\mathcal{S}$ also vanishes on $\mathcal{S}$. Joining the two ends, we infer that the regularity of the characteristics together with the appropriate dimension is equivalent to the vanishing condition for the Lagrange bracket. The reason for replacing the usual Jacobi bracket, in the integrability condition, by the Lagrange bracket, resides in the possibility, thereafter, of working locally with a system $\left\{f_{\alpha}=0\right\}$ of defining equations for the system $\mathcal{S}$.

Our results extend, to systems of partial differential equations, the classical theory of the Cauchy characteristics and the Lie construction of these characteristics for a single first order equation, in which case no compatibility conditions are required. In other terms, a single first order equation in one unknown function is always integrable whereas two or more equations are, in most cases, incompatible and consequently non-integrable. General equations involving more than one unknown function are a very delicate and yet not fully understood subject, since additional compatibility requirements must be examined, hence will not be discussed here. We show, along 
the way, how to integrate an integrable system using a simple extension of the Cauchy method.

As already mentioned, section 2 compares various brackets, has a calculatory nature and the discussion culminates with Lemma 2.1, where part of the previous calculations is placed in evidence, providing thereafter the necessary formula for proving a Theorem due to Sophus Lie.

In the section 3, we discuss higher order integral contact elements in the context of Grassmannian bundles, attention being given on how integral elements, of a certain dimension and a given order, can be extended to integral contact elements of one higher dimension. Needless to say that such an augmentation is not always possible giving rise to the concept of a maximal dimensional integral contact element. The section terminates with a Theorem, also due to Sophus Lie, that enhances the importance of the Canonical Contact Structures, defined on the Grassmannian bundles, and that will induce, by restriction, the Canonical Contact Structures associated to every differential system.

In the section 4, the local equivalence of differential equations is discussed in the realm of prolongation spaces. Local equivalence criteria are extremely useful since, as mentioned in the text, any integration method consists essentially in establishing a local equivalence of the given system of differential equations with an appropriate model. The two stated definitions of a local equivalence are due to Élie Cartan.

The section 5 provides some information on the local equivalence of various types of equations and, in the section 6 , we discuss the local equivalence of Lie groupoids, these being the defining differential systems for the Lie pseudo-groups. This latter section stands, moreover, as the presentation stage of a very rich domain where the differential systems are ever present.

The sections 7 and 8, on integral contact elements and integral submanifolds, are the starting point for the main concepts and result contained in this paper, the most enlightening ones having been conceived or at least suggested by Élie Cartan. In order to do so, he introduced, with each Pfaffian system $\mathcal{S}$, an integer that he called the character of $\mathcal{S}$ (caractère) together with the successive enlarged characters. The section 7 is devoted to the construction of these characters.

In the section 9 we introduce the derived system associated to a given Pfaffian system this being a new Pfaffian system of smaller rank. The successively iterated derived systems constitute one of the most important sequences associated to a Pfaffian system and enable, inclusively, the detection of the maximal integral sub-manifolds of the given system.

The remaining sections are devoted, on the one hand, to examine Pfaffian systems with characters equal to one or two and, on the other, to exhibit on 
specific though far from trivial types of differential systems, the integration procedure or integration method conceived by Sophus Lie which is based on successive reductions followed by lift ups that can be executed locally on a given differential system once the choice of a Jordan-Hölder Resolution, for the pseudo-group of all the local automorphisms of the system, has been made. One might even improve the Lie method by just operating with a sub-pseudogroup of the above mentioned pseudo-group since, on many occasions, such pseudo-groups do appear spontaneously within a given context.

In the section 14, we shall find a Theorem, due to Élie Cartan, that specifies the rank of the derived system associated to a Pfaffian system with character equal to two as well as a Theorem, the main contribution of this paper, stating that the exceptional situation occurring in Cartan's statement cannot be present when the Pfaffian system has null characteristics. Finally, the last section is devoted to some examples as well as to a remembrance of a beautiful paper written by Élie Cartan on Differential Galois Theory.

\section{BRACKETS}

Given an odd classical contact structure $\mathcal{P}$ on a manifold $M$ of dimension $2 n+1$, we know that the defining Pfaffian system is locally generated by a 1 -form $\omega$ with Darboux class equal to $2 n+1$. The generator $\omega$ can be chosen globally (i.e., with domain equal to $M$ ) if and only if $M$ is orientable. The pair $(M, \omega)$, where $\omega$ is global and of constant Darboux class equal to $\operatorname{dim} M$, is known as a Pfaffian structure and it induces, of course, a contact structure $\mathcal{P}$. We derive, in this section, some results concerning Pfaffian structures and introduce, in particular, the associated Jacobi and Poisson brackets. When global generators are not available, as is the case for the canonical contact structure on the Grassmannian $G(M)$, these results will be applied on the domains of the local generators $\omega$.

Let $(M, \omega)$ be a Pfaffian structure and $\mathcal{P}$ the induced contact structure. Since the dimension of the characteristic system $\Delta$ of $d \omega$ is equal to 1 (i.e., $\operatorname{dim} \Delta_{x}=1$ ), there exists a canonically defined vector field $\xi_{0}$ satisfying the equations

$$
i\left(\xi_{0}\right) d \omega=0 \quad i\left(\xi_{0}\right) \omega=1 .
$$

This vector field is an infinitesimal automorphism of $\omega$ i.e., $\theta\left(\xi_{0}\right) \omega=0$ hence, a fortiori, an automorphism of $\mathcal{P}$ namely, the Lie vector field with hamiltonian equal to the constant function 1 . The distribution $\Sigma=\operatorname{ker} \omega$ and the characteristic distribution $\Delta$ of $d \omega$ define a direct sum decomposition $T M=\Delta \oplus \Sigma$. Denoting by $\pi_{\Sigma}$ the projection upon the factor $\Sigma$, any 
vector field $\xi$ on $M$ can be decomposed according to the formula

$$
\xi=f \xi_{0}+\pi_{\Sigma} \xi
$$

where $f=i(\xi) \omega$. In particular, $\xi$ is a Lie vector field if and only if

$$
\left(d f+i\left(\pi_{\Sigma} \xi\right) d \omega\right) \wedge \omega=0
$$

and, this being the case,

$$
d f+i\left(\pi_{\Sigma} \xi\right) d \omega=\left(i\left(\xi_{0}\right) d f\right) \omega .
$$

Furthermore, the vector field $\xi$ is an infinitesimal automorphism of $\omega$ if and only if $i\left(\xi_{0}\right) d f=0$ hence, if and only if the function $f$ is a first integral of the characteristic distribution $\Delta$. Denote by $\mu$ the linear map that assigns to each vector field $\eta$ tangent to $\Sigma\left(i . e ., \eta_{x} \in \Sigma_{x}\right)$ the Pfaffian form $i(\eta) d \omega$.

Since $i\left(\xi_{0}\right)[i(\eta) d \omega]=0$, we infer that $\mu$ establishes an isomorphism between the module of vector fields tangent to $\Sigma$ and the module of 1-forms vanishing on the characteristic distribution $\Delta$. A simple calculation provides the formula

$$
\xi=f \xi_{0}+\mu^{-1}\left(\left\langle\xi_{0}, d f\right\rangle \omega-d f\right),
$$

for any Lie vector field $\xi$, where $f$ is the hamiltonian of $\xi$. In this particular setting, the Lagrange bracket is given by

$$
[f, g]=i(\xi) d g-g i\left(\xi_{0}\right) d f
$$

where $\xi$ is the Lie vector field with hamiltonian $f$. Introducing the Jacobi bracket

$$
\{f, g\}=i(\xi) d g-f i\left(\xi_{0}\right) d g
$$

the formula above reduces to the more symmetrical expression

$$
[f, g]=\{f, g\}+f i\left(\xi_{0}\right) d g-g i\left(\xi_{0}\right) d f,
$$

and it comes out that

$$
\{f, g\}=d \omega(\xi, \eta)
$$

where $\eta$ is the Lie vector field with hamiltonian $g$ or, equivalently,

$$
\{f, g\} \omega \wedge(d \omega)^{n}=n d f \wedge d g \wedge \omega \wedge(d \omega)^{n-1} .
$$

In adapted local coordinates $\left(\mathcal{U} ; x^{i}, y, p_{i}\right)$ i.e., coordinates reducing $\omega$ to its canonical form $\omega=d y-p_{i} d x^{i}$, we obtain the expressions:

$$
\begin{gathered}
\{f, g\}=\sum \frac{\partial f}{\partial p_{i}}\left(\frac{\partial g}{\partial x^{i}}+p_{i} \frac{\partial g}{\partial y}\right)-\sum \frac{\partial g}{\partial p_{i}}\left(\frac{\partial f}{\partial x^{i}}+p_{i} \frac{\partial f}{\partial y}\right), \\
\xi_{0}=\partial / \partial y, \quad i\left(\xi_{0}\right) d g=\partial g / \partial y .
\end{gathered}
$$

It is useful to remark, as a consequence of the expression (2.2), that the Lagrange bracket $[f, g]$ reduces to the Jacobi bracket $\{f, g\}$ on the subvariety defined by the equations $f=g=0$ or whenever $f$ and $g$ are first 
integrals of the characteristic system $\Delta$. In the later case, $\frac{\partial f}{\partial y}=\frac{\partial g}{\partial y}=0$ and the local expression (2.3) shows that the Jacobi bracket is simply the Poisson bracket with respect to the 2-form $d \omega$.

Geometrically speaking, this can be described as follows. The characteristic system $\Delta$ of $d \omega$ being integrable, we can consider, locally, the quotient manifold $\bar{M}$ of $M$, modulo the characteristics, factor $d \omega$ to a closed 2-form $\overline{d w}$ of maximum rank on $\bar{M}$ and a first integral $f$ to a function $\bar{f}$.

We next recall, $c f$. Part I, [18], that a Lie vector field $\xi$ with hamiltonian $f$ is an infinitesimal automorphism of $\omega$ if and only if the function $f$ is a first integral of $\Delta$. Such a vector field $\xi$ preserving $\omega$ will also preserve $d \omega$, thereafter preserving as well the characteristic system of $d \omega$. We infer that $\xi$ factors to a vector field $\bar{\xi}$ defined on $\bar{M}$ and the relation

$$
d f+i\left(\pi_{\Sigma} \xi\right) d \omega=0
$$

implies that $d \bar{f}=-i(\bar{\xi}) \overline{d \omega}$ hence $\bar{\xi}$ is a hamiltonian vector field with symplectic hamiltonian $\bar{f}$. The Poisson bracket $(f, g)$, relative to $d \omega$, factors to the usual Poisson bracket $(\bar{f}, \bar{g})$ with respect to the symplectic form $\overline{d \omega}$.

Let us finally consider a Lie vector field $\xi$ with (contact) hamiltonian $f$ and let $g$ be a generic function on the manifold $M$. According to the formula (2.1),

$$
\{f, g\}=\left(\xi-f \xi_{0}\right) g,
$$

hence the adjoint action $g \mapsto\{f, g\}$, relative to a fixed function $f$, is equal to the action of the vector field $\xi_{f}=\xi-f \xi_{0}$.

Lemma 2.1. Let $\xi$ and $\eta$ be two Lie vector fields, defined on the manifold $M$, with hamiltonians $f$ and $g$ respectively and let us assume that $\{f, g\}_{x}=0$ at some point $x \in M$. Then $[\xi, \eta]_{x}$ is a linear combination of the vectors $\xi_{x}$ and $\eta_{x}$ that, translated in terms of coordinates, yields the relation

$$
[\xi, \eta]_{x}=(\partial g / \partial y)_{x} \xi_{x}-(\partial f / \partial y)_{x} \eta_{x} .
$$

We can now derive a classical result, due to Sophus Lie (see the references), concerning the integrability of complete systems.

Theorem 2.2 (Lie). Let $\left(f_{i}\right), 1 \leq i \leq q$, be a family of functions defined on the manifold $M$ and satisfying the Jacobi bracket condition $\left\{f_{i}, f_{j}\right\}=0$ for all the indices $i$ and $j$. Then the system of linear partial differential equations

$$
\left\{f_{1}, g\right\}=0, \cdots,\left\{f_{q}, g\right\}=0,
$$

in the unknown function $g$ is complete. 
To say that the system is complete means that the (eventually singular) distribution generated by the vector fields $\bar{\xi}_{i}=\xi_{i}-f_{i} \xi_{0}$ is integrable, $\xi_{i}$ being the Lie vector field with hamiltonian $f_{i}$ and $\xi_{0}$ the vector field naturally associated to the canonical contact structure, as defined previously, and whose hamiltonian is equal to 1 . When the Lie vector fields $\xi_{i}$ generate, at each point $x \in M$, a linear sub-space transverse to $\xi_{0}$, then the linear independence of the vector fields $\bar{\xi}_{i}$ is equivalent to that of the $\xi_{i}$. We can therefore apply the criterion provided by the [18, Lemma 7.5].

Assuming now that the vector fields $\bar{\xi}_{i}$ are independent, the completeness, in the sense given to it by Sophus Lie, means the existence of $\operatorname{dim} M-q$ independent solutions $g_{j}$ of the system $\left\{f_{i}, g\right\}=0,1 \leq i \leq q$.

\section{Higher order DifFerential EQUations}

Let $M$ be a manifold of dimension $n, x_{0} \in M$ a given though arbitrary point and $\left(N_{1}, N_{2}\right)$ a couple of sub-manifolds of $M$ both containing the point $x_{0}$ and both having their dimensions equal to $q$. We say that the two sub-manifolds have a contact of order $k, k \geq 0$, at the point $x_{0}$ when there exists a local diffeomorphism

$$
\varphi: U_{1} \subset N_{1} \rightarrow U_{2} \subset N_{2},
$$

defined on an open neighborhood $U_{1}$ of the point $x_{0}$ and having as target an open neighborhood $U_{2}$ of the same point, such that the two mappings $\iota_{1}$ and $\iota_{2} \circ \varphi$ have a contact of order $k$ at the point $x_{0}$, where $\iota_{i}$ denotes the inclusion map.

Stated more explicitly in terms of $k$-jets, the above contact requirement simply means that $j_{k} \iota_{1}\left(x_{0}\right)=j_{k} \iota_{2} \circ \varphi\left(x_{0}\right)$, the notation $j_{k} f(x)$ indicating the $k$-jet of the mapping $f$ taken at the point $x$.

The above defined relation is, of course, an equivalence relation, the equivalence classes being called the $k$-th order tangent contact elements to the manifold $M$. Further details and results on this subject can be found in [23].

The set of all the $k$-th order contact elements is endowed with a structure of a differentiable manifold, denoted by $G_{k}^{q}(M)$, and the "base point" map

$$
\pi: G_{k}^{q}(M) \rightarrow M
$$

is a locally trivial fibre bundle. First order contact elements are simply $q$-dimensional linear sub-spaces contained in the tangent spaces to the manifold $M$ hence, in particular, $G(M)=G_{1}^{n-1}(M)$, where $n=\operatorname{dim} M$.

We observe that $G_{k}^{q}(M)$, also known as the $k$-th order Grassmann bundle, admits an everywhere dense open subset namely, the bundle of all the $k$-jets of local sections of a fibration (submersive surjection) $M \rightarrow M_{0}$. For this, it suffices to map the $k$-th order jet $j_{k} \sigma(y)$ of a local section $\sigma$, at the point 
$y \in M_{0}$, into the $k$-th order equivalence class of the sub-manifold im $\sigma$ at the point $x=\sigma(y)$. We list below some useful properties verified by these higher order contact elements.

(a) For any $r<q$, every $r$-dimensional contact element of order $k$ extends to a not necessarily unique $q$-dimensional contact element of the same order $k$.

(b) For any $h<k$, every $h$-th order contact element of dimension $q$ extends to a not necessarily unique $k$-th order contact element of the same dimension $q$.

(c) We can also combine and iterate the above two extension procedures and the concept of inclusion as well as that of iteration, for contact elements, is not only well defined but has moreover a wide scope of possibilities.

(d) Any local diffeomorphism $\varphi: U \rightarrow U^{\prime}$ of $M$ extends (prolongs) canonically to a local diffeomorphism $\wp \varphi: \mathcal{U} \rightarrow \mathcal{U}^{\prime}$ of the Grassmann bundle $G_{k}^{q}(M)$ and, actually, this prolongation procedure can also be carried out for any local diffeomorphism $\varphi$ initiating on a manifold $M$ and terminating on a different manifold $M^{\prime}$.

Let us next define the $k$-th order canonical contact structure namely, a Pfaffian system on the manifold $G_{k}^{q}(M)$ that will play a fundamental role in the study of the integrability problem for $k$-th order partial differential equations. We call holonomic the contact elements as defined earlier and introduce now a notion of semi-holonomy.

Given a $q$-dimensional sub-manifold $N \subset M$ and fixing a point $x_{0} \in N$, let us denote by $H_{k, x_{0}} \in\left(G_{k}^{q}\right)_{x_{0}}$ the $k$-th order contact element determined by the above data. Considering the sub-manifold $N_{k-1} \subset G_{k-1}^{q}$ and, subsequently, taking the first order contact element determined by this sub-manifold at the corresponding point $H_{k-1, x_{0}}$, let us indicate by $H_{1, H_{k-1, x_{0}}}$ the corresponding first order contact element thus obtained and call it semi-holonomic. This element is clearly well determined since the initially given contact element provides all the necessary derivatives up to the order $k$ in order to be so. We thus obtain a map

$$
\operatorname{sh}_{k, k-1}: G_{k}^{q}(M) \rightarrow G_{1}^{q}\left(G_{k-1}^{q}(M)\right)
$$

that we call the semi-holonomic inclusion and a similar procedure also provides the more general semi-holonomic inclusion

$$
s h_{k, k-h}: G_{k}^{q}(M) \rightarrow G_{h}^{q}\left(G_{k-h}^{q}(M)\right) .
$$

We can now introduce the canonical contact structure of order 1 , on the manifold $G_{k}^{q}$, by proceeding recurrently on the order $k$. For $k=1$, define 
$\mathcal{P}=\mathcal{P}_{1}$ by setting $\mathcal{P}_{H_{x}}=\left(T \pi_{H_{x}}\right)^{*}\left(H_{x}\right)^{\perp}$ and in general, for $\mathcal{P}=\mathcal{P}_{k}$,

$$
\mathcal{P}_{H_{k, x}}=\left(T \rho_{k, k-1}\right)^{*}\left(s h_{k, k-1}\left(H_{k, x}\right)\right)^{\perp}+\left(T \rho_{k, k-1}\right)^{*} \mathcal{P}_{k-1, H_{k-1, x}},
$$

where $\rho_{k, h}$ denotes the natural projection of the $k$-contact elements onto $h$-contacts and the tangent maps are taken at the appropriate points. The main properties of these Pfaffian systems can be stated in the following theorem, where $N_{k}$ denotes the sub-manifold of $G_{k}^{q}(M)$ obtained by taking all the $k$-th order contact elements defined by the sub-manifold $N \subset M$.

Theorem 3.1 (Lie). The following two properties describe the basic attributes and importance of the canonical contact structures at any orders:

(a) A sub-manifold $\bar{N} \subset G_{k}^{q}(M)$ is of the form $N_{k}$ if and only if it is an integral manifold of $\mathcal{P}_{k}$ transversal to the projection $\pi$ onto $M$.

(b) A local diffeomorphism $\bar{\varphi}$ of $G_{k}^{q}(M)$ is of the prolonged form $\wp \varphi$ if and only if it is projectable onto a local diffeomorphism $\varphi$ on $M$ and if, moreover, it is an automorphism of the contact structure $\mathcal{P}_{k}$.

Similar properties are, of course, true as well for the infinitesimal automorphisms.

We denote, as previously, by $\mathcal{P} \mid \mathcal{S}$ or $\mathcal{P}_{k} \mid \mathcal{S}$ the restriction of the contact system to a differential equation $\mathcal{S}$ of order $k$ and adopt as well the notation $\mathcal{N}_{k}$ to indicate the manifold composed by all the $k$-th order contact elements determined by the sub-manifold $\mathcal{N}$ of $M$. This being so, we can now reformulate all the results of the previous sections in terms of the differential equations of order $k$. However, we shall not enter here into the details since these would be essentially a copy of what is already written, the index 1 being replaced by $k$.

\section{The local equivalence PRoblem}

We discuss here the equivalence problem in the realm of Ehresmann's prolongation spaces of which Jet spaces and Grassmannian contact bundles are special cases, [10],[11], [12]. All the emphasis is given to the first order canonical contact systems associated to the partial differential equations since the local or, inasmuch, the global equivalence of these Pfaffian systems will entail the corresponding equivalence for the equations. A fundamental ingredient in our approach is the consideration of merihedric prolongation spaces (prolongements mériédriques) as defined and used by Élie Cartan in many of his writings.

Though rather absent in the recent literature, the reader will find interesting examples in [14], [19] and [17]. As for the calculations, we try to 
reduce them to the strict minimum, the main concern being the determination of a fundamental set of invariants associated to a differential system that will characterise as well as describe the local equivalences.

Let us recall, briefly, some very standard facts so as to fix the notations and subsequently discuss, in more detail, the aims and techniques of the next two sections.

As is usual, a fibration is a surjective map $P \rightarrow M$ of maximum rank and we define a multi-fibration as being a sequence, finite or infinite in length,

$$
\cdots \rightarrow P_{\mu} \rightarrow P_{\mu-1} \rightarrow \cdots \rightarrow P_{1} \rightarrow P_{0}
$$

where all the projections $\rho_{\beta, \alpha}: P_{\alpha} \rightarrow P_{\beta}, \alpha \geq \beta$, are fibrations, the map $\rho_{\beta, \alpha}$ being the composite of the successive projections.

Following Cartan, we say that a multi-fibration is a merihedric prolongation space with respect to the basic manifold $P_{0}$ when there exists a prolongation algorithm $\wp$ such that any local transformation $\varphi: U \rightarrow V$ on the manifold $P_{0}$ can be prolonged (extended) to a local transformation $\varphi_{\mu}=\wp_{\mu}(\varphi)$ operating on the manifold $P_{\mu}$, the usual commutativity relations with respect to the projections as well as to the composition of transformations and the passage to the inverses being preserved.

However, we shall only require, with the obvious notations, that the open sets $U_{\mu}$ and $V_{\mu}$ project surjectively onto $U_{\nu}$ and $V_{\nu}$ whenever $\nu \leq \mu$ without requiring that the former be the inverse images of the latter. We next require that to every local sub-manifold $\mathcal{N}$ of $P_{0}$ corresponds, at each level $\mu$, a prolonged sub-manifold $\wp \mathcal{N}$ and that these sub-manifolds project one upon the other. Finally we require that, at each level $P_{\mu}, \mu \geq 1$, a Pfaffian system $\mathcal{C}_{\mu}$ be defined and that it satisfies the following properties:

(a) $\rho_{\mu, \mu-1}^{*} \mathcal{C}_{\mu-1} \subset \mathcal{C}_{\mu}$,

(b) for any local sub-manifold $\mathcal{N}$ of $P_{0}$, the space $\wp_{\mu} \mathcal{N}$ is an integral submanifold of $\mathcal{C}_{\mu}$ and, conversely, any local sub-manifold $\overline{\mathcal{N}}$ of $P_{\mu}$ that is transverse to the fibres over $P_{0}$ and is integral for the previous system be, locally, the prolongation of a sub-manifold in the base space and

(c) the $\mu$-th prolongation of any local transformation of $P_{0}$ is an automorphism of $\mathcal{C}_{\mu}$, the converse being verified just locally.

A similar statement holds for vector fields (infinitesimal transformations) and their prolongations, where the operations are now the linear operations together with the Lie derivative and the bracket of vector fields. We also require that this infinitesimal prolongation procedure be compatible with the finite prolongation of the elements of a local 1-parameter group. 
Besides the Grassmannians, among the most requested prolongation spaces are, of course, the sequences of Ehresmann's jet spaces of all orders associated to given fibrations $\pi: P \rightarrow M$, where we consider $k$-jets of local sections. We do not provide any details on jet spaces since these are a well known subject.

Let us finally indicate a prolongation procedure for differential equations i.e., sub-manifolds $\mathcal{S}$ contained in merihedric prolongation spaces by adopting exactly the same definitions as those given in the case of Jet spaces. In fact, let us take a sub-manifold $N \subset P_{0}$ with its dimension equal to that prescribed for the solutions of a given equation $\mathcal{S} \subset P_{\mu}$ and say that it is $\ell$-admissible, at the point $x \in N$, when it satisfies the following two properties:

(a) $\wp_{\mu}(N)_{x} \in \mathcal{S}$, where the index $x$ indicates the point of $\wp_{\mu}(N)$ that corresponds to $x$ and, at that point,

(b) the sub-manifold $\wp_{\mu}(N)$ is tangent up to order $\ell$ to $\mathcal{S}$.

We define, thereafter, the $\ell$-th prolongation $\wp_{\ell}(\mathcal{S})$ of $\mathcal{S}$ as being the totality of the $(\mu+\ell)$-th order contact elements obtained by means of the $\ell$-admissible sub-manifolds and calculated at the admissibility points. All the properties of the standard prolongation algorithm for differential equations, defined in Jet spaces, transcribe in this more general setting and, in particular, the $\ell$-th prolongation of $\mathcal{S}$ can be obtained by iterated first order prolongations.

In studying the equivalence, local or global, of two differential equations, we shall always consider both equations defined over the same basic manifold for, if the two equations were situated above distinct basic manifolds, we could always shift one onto the other by taking a local or global diffeomorphism whose prolongations will also transport all the other data. Concerning the basic properties and operations relative to differential equations and their prolongations, we refer the reader to [22].

Let us now begin by examining the notion of equivalence. This notion must respect the solutions of the equations i.e., a solution of one of them must be transformed into a solution of the other hence must respect the sophisticated structure of the Grassmannian spaces.

Definition 4.1. Two $k$-th order differential equations $\mathcal{S}$ and $\mathcal{S}^{\prime}$ are said to be locally absolutely equivalent in the neighborhoods of $X \in \mathcal{S}$ and $X^{\prime} \in \mathcal{S}^{\prime}$ when there exists a local transformation $\varphi: U \rightarrow U^{\prime}$ on the total manifold $\mathrm{P}$ such that its prolongation $\wp_{k} \varphi$ transforms $\mathrm{X}$ into $\mathrm{X}^{\prime}$ and, further, becomes a diffeomorphism when restricted to the appropriate open subsets of $\mathcal{S}$ and $\mathcal{S}^{\prime}$. The equivalence is global when $\varphi$ is global. 
Obviously, $\wp_{k} \varphi^{-1}$ is also an equivalence and any one of them transforms solutions into solutions. If, for instance, $\mathcal{N}$ is a solution of $\mathcal{S}$, then $\varphi(\mathcal{N})$ is a solution of $\mathcal{S}^{\prime}$.

The notion of absolute equivalence seems too restrictive when confronted with applications and we now give, below, a broader and more suitable definition. Needless to say that both definitions are stated in Cartan's Mémoire [6].

Definition 4.2. Two differential equations are said to be locally equivalent when they admit prolongations of certain orders that are locally absolutely equivalent. Global equivalence has a similar definition.

Most probably, the reader already observed that the last definition is a big cheat. In fact, it is so since the absolute equivalence of some prolongations implies, by projection, the absolute equivalence of the given equations. Nevertheless, quite often it is easier and more convenient to deal with prolongations than with the given equations. Unfortunately, Cartan is not here to give us a more convincing argument.

The first example occurs in the case of ordinary differential equations. In this case, when the image spaces have the same dimension (the same number of dependent variables), any two equations are always locally equivalent at non-singular points. When singularities are present, the matter becomes considerably more involved.

As a second example, we mention that all the integrable Pfaffian systems with the same rank and co-rank are locally equivalent. As for the global equivalence, in the present case as well as in the case of ordinary differential equations, this is a beautiful and still unresolved problem in topology. A very interesting situation can be found in [1].

Let us now have a glance at non-integrable Pfaffian systems, a rather nice example being provided by the Flag Systems. These are systems of co-rank equal to 2 (when the characteristics vanish) hence the integral manifolds are just 1-dimensional curves, l'intégrale générale ne dépendant que de fonctions arbitraires d'une seule variable, as would claim Cartan. However, the reader might delight himself in examining the many local models exhibited in [19].

These Flag Systems provide a very nice illustration of the merihedric context and, further, are prolongation spaces of finite length. Before doing so, we recall ([14]) that, in jet spaces, the total derivative of functions can be trivially extended to a total Lie derivative of differential forms and, with this in mind, we shall give attention to Pfaffian systems instead of partial differential equations, which was by far Cartan's preference. In this respect, we also recall that the total Lie derivative of the $k$-th order canonical contact structure, defined on a Jet space $J_{k} \pi$, is precisely the corresponding contact 
system on the $(k+1)$-st level (where we have to add, for obvious reasons, the pullback of the contact structure defined on $J_{1} \pi$ ). Let us then return to the sequence

$$
\left(P_{\ell}, S\right) \rightarrow\left(P_{\ell-1}, \bar{S}_{1}\right) \rightarrow \cdots \rightarrow\left(P_{0}, \bar{S}_{\ell}\right),
$$

exhibited in [19, p.8, 1.-3], $S$ being a Flag System of length $\ell$ defined on the space $P=P_{\ell}$, with null characteristics and where $\bar{S}_{\ell-\nu}$ denotes a Pfaffian system canonically isomorphic to a successive derived system of $S$ though defined on a more appropriate spaces so as to also display null characteristics.

The above finite sequence is a prolongation space together with Pfaffian systems, at each level, naturally associated to the initially given system $S$. The terminating system $\bar{S}_{\ell}$ vanishes and $\bar{S}_{\ell-1}$ is a Darboux system in 3-space. As shown in [19], every local or infinitesimal automorphism of this Darboux system extends (prolongs) canonically to an automorphism of $\left(P_{\ell-\nu}, \bar{S}_{\nu}\right)$, this correspondence becoming, moreover, an isomorphism of pseudo-groups as well as of pseudo-algebras. We are not to be concerned with local sections since $P_{1}$ can be considered as the total space, whereas the base space $P_{0}$ collapses to an open set, eventually to a point.

We claim that each Pfaffian system $\bar{S}_{\nu}$ is the prolongation of $\bar{S}_{\nu+1}$. As is, not only it seems that we are walking backwards but worse, the prolongation algorithm as described previously is somehow missing. Nevertheless, in the passage from $\bar{S}_{\nu+1}$ to $\bar{S}_{\nu}$, three options are possible, [21], and, upon choosing the appropriate one, we can thereafter apply the prolongation method as described earlier. Furthermore, if we choose the appropriate coordinates so as that the form $\omega=d y-z d x$ generates the Darboux system $\bar{S}_{\ell-1}$, then total derivatives reappear again and Cartan, as always, is perfectly right in his claims.

\section{The LOCAL EQUiVALENCE OF DIFFERENTIAL SYSTEMS}

We shall now examine a few aspects of this local equivalence problem where most enlightening examples are provided by the study of geometrical structures since, in most cases, the local equivalence is examined on the level of the defining equations for these structures ([15]).

(a) Ordinary Differential Equations. An ordinary differential equation of order 1 in $n$ unknown functions is simply a vector field on a manifold $P$, where we take the fibration $\pi: P \rightarrow \mathbf{R}$ with $\operatorname{dim} P=n+1$. A $k$ th order equation is a contact vector field (infinitesimal automorphism of the corresponding contact structure) defined on $J_{k} \pi$. As mentioned earlier, any two ordinary differential equations of the same order are always locally equivalent at two non-singular points. As for the global equivalence, this is 
a topological problem that involves the nature of the global solutions and, of course, compactness is the main ingredient. We also observe that two ordinary differential equations of distinct orders can be locally, viz. globally, equivalent and Cartan's definitions start to make much more sense. As for the local equivalence in the neighborhoods of singular points, there is an immense literature on this subject hence we shall not enter here into details concerning this matter.

(b) Linear Partial Differential Equations. Such equations are defined, most conveniently, as vector sub-bundles of the $k$-th order Jet spaces of local sections of vector bundles i.e., the total space of the initial fibration is the total space of a vector bundle and, consequently, the corresponding Jet space is as well a vector bundle. In this case, it is most appropriate to restrict the local equivalences to linear morphisms though two linear equations can actually be equivalent via a non-linear local transformation. This is not a flaw but an immense virtue since it enables us to replace a non-linear equation by a linear one. A first systematic investigation on the local equivalence of linear differential systems was initiated by Drach, Picard and Vessiot, whereupon it resulted in the well known Théorie de Picard-Vessiot (cf. [16]).

(c) General Differential Equations. Who undoubtedly most contributed not only with results but mainly with ideas and methods, in this general equivalence problem, was Élie Cartan and the reader is invited to examine the table of contents of the Oeuvres Complètes, Partie II. Very significant contributions were also brought by Bernard Malgrange, in particular for the case of elliptic equations, as well as by Masatake Kuranishi for the case of the defining equations of a Lie pseudo-group namely, those equations that have the additional algebraic structure of a groupoid. Here again, we shall not enter into further details since the subject is by far too extensive.

(d) Pfaffian Systems. Initial contributions were, of course, brought by Pfaff himself and later expanded by contemporary Sophus Lie (Gasammelte Abhandlungen, Vol. 6) and Mark von Weber. But again, the most striking contributions were placed forward by no other than Élie Cartan who gave us much insight in the local equivalence of the non-integrable systems. As mentioned earlier, any two integrable Pfaffian systems are everywhere locally equivalent if and only if they have the same rank and co-rank (the underlying manifolds ought to have the same dimension). It is also a straightforward consequence of linearity in the tangent and co-tangent spaces that any two Pfaffian systems, integrable or not, are equivalent, to first order, if and only if their ranks and co-ranks are equal. 
(e) Exterior Differential Systems. All the merits belong here to Élie Cartan who, in fact, introduced such systems in the study of various geometrical problems, [8]. Nevertheless, important contributions are also due to Masatake Kuranishi.

\section{The LOCAL EQUivalence of LIE GROUPOIDS}

Since we are interested in the local equivalence of partial differential equations, the sole Lie groupoids to be considered in the sequel are those whose total spaces are sub-manifolds in some jet space and we begin here with some general considerations.

Given the fibration $\pi=p_{1}: P=M \times M \rightarrow M$ and the corresponding $k$-th order Jet bundle $J_{k} \pi$, we denote by $\Pi_{k} M$ the groupoid of all the $k$-jets of invertible sections of $\pi$, [17]. In other terms, $\Pi_{k} M$ is the set of all the invertible $k$-jets with source and target in $M$ and is an open dense subset of $J_{k} \pi$.

The Lie groupoids we shall be interested in are the sub-groupoids of $\Pi_{k} M$ that are inasmuch sub-manifolds (not necessarily regularly embedded) of the ambient groupoid and, together with the differentiable sub-manifold sructure, become Lie groupoids in the sense of Ehresmann, cf. [10], [11], and [12]. Such $k$-th order Lie groupoids will, most often, be denoted by $\Gamma_{k}$.

We next observe that the standard prolongation of order $h$ of a Lie groupoid, in the sense of partial differential equations, is again a Lie groupoid at order $k+h$ and all the usual properties relative to differential equations transcribe for these Lie groupoids. Moreover, it can be shown that the above-mentioned properties still hold in the more general context of merihedric prolongations. In considering the local equivalences, we proceed in analogy with what is usually done in Lie group theory and restrict our attention to open neighborhoods of the unit elements of the groupoids.

More precisely, we look for conditions under which there exists a local contact transformation that maps isomorphically an open neighborhood of the unit elements in one of the groupoids onto a similar neighborhood of the other. The term "isomorphically" means that it preserves the algebraic as well as the differentiable properties and "an open neighborhood of the unit elements" refers to a neighborhood of an open set in the units sub-manifold.

Since a contact transformation, as above, operating on the sub-manifolds is by necessity induced by a local contact transformation operating in the ambient Jet space, we ultimately consider, in our setting, a local contact transformation $\varphi: \mathcal{U} \rightarrow \mathcal{U}^{\prime}$ that transforms accordingly the respective intersections with the groupoids and where $\mathcal{U}$ and $\mathcal{U}^{\prime}$ are open sets in $\Pi_{k} M$.

We begin by considering the equation $\mathcal{R}$ composed by all the elements $X \in \Pi_{k} M$ whose sources belong to the units of the first groupoid and the 
targets to the units of the second. Then, of course, any local equivalence will be a local solution of this equation, the converse being also true as soon as the appropriate tansversality conditions, with respect to the $\alpha$-fibres, are respected and where a local transformation of the base space operates on $\Pi_{k} M$ via the conjugation by its $k$-jets.

Let us next observe that the first groupoid operates, to the right, on the space $\mathcal{R}$ and that the second groupoid operates to the left. So as to render the data more homogeneous and regular, we shall assume that both these actions are transitive, such an assumptions being much in accordance with the problem considered.

In fact, most relevant examples do conform to this requirement and, more important, most contexts where equivalence is sought for also do comply with it. It should be noted that whenever the local equivalence is verified and a local contact transformation is put forward, then one transitivity assumption gives rise to the other.

It now remains to investigate the equation $\mathcal{R}$ as well as its prolongations and determine the desired invariants that will characterize the local equivalence. A first obvious remark is that the dimensions of both groupoids inasmuch as the dimensions of their units sub-spaces must be equal and this also entails the equality, in dimensions, of both the $\alpha$ and the $\beta$ fibres. The standard as well as the merihedric prolongation algorithms do preserve some data and operations but not all. In fact, the prolongation of a groupoid is still, algebraically, a groupoid but its differentiable structure is not guaranteed. Furthermore, the action of the prolonged groupoid on the prolonged equation is not forcibly transitive. On the other hand and concerning the equation $\mathcal{R}$, similar statements can also be affirmed. Nevertheless, it should be emphasized that the above stated transitivity assumption implies that this equation inherits, from either groupoid, a manifold structure rendering it a sub-manifold of $\Pi_{k} M$.

In what follows, we list several necessary conditions for our problem to have a solution.

1. Differentiability. The iterated prolongations of both groupoids are also Lie groupoids and sub-manifolds of the respective jet spaces.

2. Dimension. Both prolonged groupoids have, at each order, the same dimension. We do not need to bother about the dimensions of the unit spaces since these spaces are equal (diffeomorphic) to those of the initially given groupoids.

3. Transitivity. Both prolonged groupoids operate, at each order, transitively on the prolonged equation. Consequently, each prolonged equation is a sub-manifold of the corresponding jet space. 
4. Symbols. The symbols of both prolonged groupoids have, at each order, the same dimension.

5. $\delta$-cohomology. Both iterated prolongations of the groupoids have equidimensional $\delta$-cohomologies and thereafter become simultaneously 2acyclic, $c f .[22]$.

We now assume that the above five properties do hold for the given two Lie groupoids of jets. On account of the transitivity of the groupoid actions, we infer that the iterated prolongations of the equation $\mathcal{R}$ become also 2acyclic at orders not greater than those for the groupoids. Finally, if we assume further that all the data is real analytic, then the Cartan Theorem ${ }^{1}$ guarantees the desired local equivalence. Unfortunately, we cannot make the same statement in the $C^{\infty}$ realm since, under these weaker assumptions, the whole Cartan Theory breaks down. There are examples of non-analytic though involutive (2-acyclic) equations that do not possess any solution. In this last realm, much further work is awaiting to be done.

The local equivalence of analytic Lie groupoids entails the local equivalence of Lie pseudo-groups since these are defined with the help of such groupoids, their defining equations.

\section{INTEGRAL CONTACT ELEMENTS}

Let $\mathcal{P}$ be a not necessarily integrable Pfaffian system of constant rank $r$ defined on the manifold $M$ of dimension $n$, denote by $\left\{\omega^{1}, \omega^{2}, \cdots, \omega^{r}\right\}$ a free system of local generators, defined in a neighborhood of a generic point $x_{0}$ and by $\Sigma$ the annihilator of $\mathcal{P}$ in $T M$, thus obtaining a field of $(n-r)$-dimensional first order contact elements annihilated by $\mathcal{P}$.

Let us take an arbitrary point $x_{0} \in M$ and try to determine, de proche en proche as would say Cartan, an ascending chain of integral contact elements at the point $x_{0}$. We firstly take a one-dimensional subspace $l \subset \Sigma_{x_{0}}$, a line in the terminology of Cartan, and examine when such a linear subspace can be included in a higher dimensional integral contact element. The sole condition $l \subset \Sigma_{x_{0}}$ is not sufficient and the entire subsequent discussion reposes on the following remark:

Given an integral sub-manifold $\mathcal{I}$ of the Pfaffian system $\mathcal{P}$ and an arbitrary local section $\omega$ of this system, the restrictions $\iota^{*} \omega$ and $\iota^{*} d \omega$ vanish identically on $\mathcal{I}$, where $\iota: \mathcal{I} \hookrightarrow M$ is the inclusion.

It suffices, of course, to check the above condition for the generators $\omega^{i}$ and, further, this condition transcribes by the two requirements

$$
i(v) \omega^{i}=0,(v \in l, v \neq 0), \quad i(v) d \omega^{i} \equiv 0 \bmod \left\{\omega^{j}\right\},
$$

the latter condition also rewriting by $\left(d \omega^{i}\right)_{x_{0}} \equiv 0 \bmod \left\{\omega^{j}\right\}$.

\footnotetext{
${ }^{1}$ most improperly called the Cartan-Kähler Theorem
} 
We now fix a given 1-dimensional linear integral contact element $E_{1}$ issued at the point $x_{0}$ (i.e., contained in $\Sigma_{x_{0}}$ ) and look for the conditions under which a plane contact element $E_{2}(\operatorname{dim}=2)$ containing the given linear element $E_{1}$ is also integral. In order to find such an element, it suffices to look for a vector $w \in T_{x_{0}} M$ linearly independent from $v$ and verifying the two conditions

$$
i(w) \omega^{i}=0, \quad i(w) i(v) d \omega^{i}=d \omega(v, w)=0 .
$$

We shall say that $w$ is in involution with $v$ (in Cartan's terminology, the line $\lambda$ generated by $w$ is in involution with the line $l$ ). Linearity then implies that all the vectors $w$ that are in involution with $v$ (adding, of course, the null vector) form a linear subspace $\tilde{E}_{1}$ containing all the 2-dimensional integral subspaces considered above.

Let us next fix a 2-dimensional integral contact element $E_{2}$ (a linear 2-dimensional subspace of $\tilde{E}_{1}$ ) and consider the vectors $z$ that are in involution with all the vectors belonging to $E_{2}$. It suffices, of course, to consider those vectors that are in involution, simultaneously, with those belonging to any given basis of $E_{2}$ and we shall denote by $\tilde{E}_{2}$ the linear subspace consisting of all these vectors that obviously is contained in $\tilde{E}_{1}$.

Fixing a 3 -dimensional contact element $E_{3}$ that is contained in $\tilde{E}_{2}$ and contains $E_{2}$, we can pursue this nice Origami game and construct an ascending chain $E_{0} \subset E_{1} \subset E_{2} \subset E_{3} \cdots$, each element satisfying the involutiveness condition with respect to the preceding one. We include the null space $E_{0}$ just to please Cartan where it figures, in his writings, as the one point set $E_{0}=\left\{x_{0}\right\}$. Dimensions being finite, there will be an integer $\rho_{k}$ for which the above process breaks down namely, there will not exist any other non-null vector, in involution with $E_{\rho_{k}}$, other than those already obtained previously.

Definition 7.1. According to Cartan, we shall say that the integer

$$
s_{1}=n-r-\rho_{k}
$$

is the character (caractère) of $\mathcal{P}$ at the point $x_{0}$ and relative to the above ascending chain.

Described more geometrically, the character is simply the difference between the values of $\operatorname{dim} \Sigma_{x_{0}}$ and $\operatorname{dim} E_{\rho_{k}}$.

We next describe Cartan's most ingenious method for determining these maximal integral elements.

Let us take a tangent contact element $E$ contained in some $\Sigma_{x_{0}}$ as well as a second sub-space $E^{\prime} \subset E$. The sub-space $E^{\prime}$ is said to be characteristic with respect to $E$ whenever any element of $E^{\prime}$ is in involution with every element of $E$. The sum of all the characteristic sub-spaces is then a maximal 
characteristic sub-space called the characteristic element of $E$ with respect to $\mathcal{P}$. Though $\mathcal{P}$ is assumed to have null characteristics, these characteristic elements need not be null.

Next, given any two sub-spaces $E$ and $F$ contained in $\Sigma_{x_{0}}$, we shall say that they are conjugate when their characteristic elements are in involution, this meaning that any vector belonging to one of them is in involution with all the vectors belonging to the other. In order to obtain an integral element of dimension $\mu \leq \rho_{k}$, it suffices to take $\mu$ independent vectors $\left\{v_{1}, \cdots, v_{\mu}\right\}$ for which the corresponding sub-spaces $\tilde{E}_{2, i}, 1 \leq i \leq \mu$, are pairwise conjugate and then simply take their intersection.

We next define $s_{j}=\operatorname{dim} \tilde{E}_{j}$, for $1 \leq j \leq \rho_{k}$ and at the given point $x_{0}$. Obviously,

$$
s_{1}-s_{2} \geq s_{2}-s_{3} \geq \cdots \geq s_{\rho_{k}-1}-s_{\rho_{k}}
$$

since, at each step, we have a lesser choice of vectors to look for and, furthermore, $\rho_{k}=n-r$ if and only if $\mathcal{P}$ is integrable in a neighborhood of $x_{0}$.

We should also observe that this point-wise technique is most appropriate for the study of singular Pfaffian systems in view of obtaining the maximal integral sub-manifolds whose dimensions can vary not only from point to point but, in fact, also at the same point since the maximal integral element depends upon the choice of the chain of intermediate integral elements.

Moreover, two distinct maximal integral elements of different dimensions can have a common intersection whose dimension is greater than 1. This is the case, for example, occuring in the systatical systems (systèmes systatiques, [2]) where two distinct maximal integral manifolds can have, at a point $x_{0}$, a first order tangency of dimension $\geq 2$ or even a common sub-manifold of such a dimension. The integers $s_{j}$ are called the successive enlarged characters of $\mathcal{P}$ at the point $x_{0}$.

We finally call the attention of the reader to the way Cartan embraced his thoughts. Invariably and persistently, he would always pursue the covariant trail and, in his Mémoire [2] as well as previously in [3], this shows up as soon as he describes the above-mentioned contact elements. In fact, these are depicted with the help of convenient linear combinations of the differentials $d x^{i}$ relative to a local coordinate system $\left(x^{1}, \cdots, x^{n}\right)$ defined in a neighborhood of the point $x_{0}$. In other terms, Cartan employs linear bases for the sub-spaces, in $T^{*} M$, that annihilate the contra-variant subspaces $E_{j}$ and $\tilde{E}_{j}$ considered previously ([nous] regardons $d x_{1}, d x_{2}, \cdots, d x_{r}$ comme les paramètres directeurs d'une droite issue de ce point, pg.4, l.8).

In so doing the calculations become much simpler and completely straightforward though, unfortunately, our contra-variant intuition of the geometric world around us suffers with it. 


\section{INTEGRAL SUB-MANIFOLDS}

We first observe that any Pfaffian system whith rank not exceeding $n-1$ always admits integral 1-dimensional sub-manifolds, at a given point $x_{0}$, since it suffices to integrate any local vector field $\xi$ that is annihilated by the system and that satisfies $\xi_{x_{0}} \neq 0$. The above assertion can fail for singular systems for, in this case, it might happen that $\Sigma_{x_{0}}=0$ at a given point $x_{0}$. Hence, there is in principle no integrability condition pending for the dimension 1. Secondly, we observe that the dimensions of all the previously considered contact elements are lower semi-continuous (the dimensions tend to increase) and, further, that the pertinence relation $\in$ is a closed relation. Consequently, these properties enable us to choose locally, in a neighborhood of $x_{0}$, a finite set of linearly independent vector fields $\xi_{\mu}, 1 \leq \mu \leq \rho_{k}$, such that

1. The vectors $\left\{\xi_{\mu}\left(x_{0}\right)\right\}$ generate $E_{\rho_{k}}$ and

2. The vector fields $\xi_{\mu}$ generate, in that neighborhood, an integrable distribution (field of contact elements).

We can then integrate this local distribution and obtain an integral submanifold $\mathcal{I}$ of the initially given Pfaffian system $\mathcal{P}$, this integral manifold being of maximal dimension relative to the point $x_{0}$ and to the ascending chain.

We should however observe that it is not unique relative to the point $x_{0}$ unless, of course, its dimension be equal to the co-rank of $\mathcal{P}$. When this is not the case, there are many possible choices for the chains. Since the element $E_{\rho_{k}}$ is maximal, relative to the sub-chain terminating by $E_{\rho_{k}-1}$, this element is unique, always relative to the sub-chain, though the above indicated integral sub-manifold needs not be locally unique, relative to the complete chain.

Furthermore, choosing the local vector fields $\xi_{\mu}$ in such a way that the vectors $\left\{\xi_{\mu}\left(x_{0}\right), \mu \leq j\right\}$ generate $E_{j}$, we can obtain, by successive integrations, an ascending chain of integral sub-manifolds respectively tangent to the elements of the linear chain, each one contained in the next manifold.

We finally observe that the singularities as well as the singular integral sub-manifolds can be located inasmuch as evaluated by computing the integer $\rho_{k}$ at the various points of the manifold $M$ and corresponding to the various ascending chains of integral elements.

\section{The DERIVED SYSTEMS}

Given a Pfaffian system $\mathcal{P}$, we recall that the characteristic system associated to $\mathcal{P}$ is the Pfaffian system generated by all the differential 1 -forms $\omega$ and $i(\xi) d \omega$ where $\xi$ is an arbitrary local vector field annihilated by $\mathcal{P}$ and $\omega$ an arbitrary local section of the given system. 
The contra-variant counterpart namely, the annihilator of the characteristic system is defined as follows. At each point $x \in M$, we consider the sub-space, of the tangent space, whose elements are the vectors $v$ such that $i(v) \omega=0$ and $i(w) i(v) d \omega=0$ for any vector $w$ annihilated by $\mathcal{P}$ and any local 1-form $\omega$ belonging to the system. In other terms, the above characteristic sub-space is defined as the set of all the vectors $v$ annihilated by $\mathcal{P}$ that further verify $d \omega(v, w)=0$ for any vector $w$ also annihilated by $\mathcal{P}$ and any local section $\omega$ of $\mathcal{P}$.

We denote by $\mathcal{C H}$ this characteristic system and observe that its purpose resides in (loosely speaking) finding the minimum number of independent function by means of which the initially given system can be re-written or, equivalently, in finding the local fibration with the largest dimensional fibres with respect to which the given system factors to the quotient i.e., to the base space of this fibration onto an equivalent quotient system, $c f$. [13]. Inasmuch, the derived system of $\mathcal{P}$, denoted by $\mathcal{P}_{1}$, is the Pfaffian system generated by the forms $\omega$, local sections of $\mathcal{P}$, for which $d \omega \equiv 0 \bmod \mathcal{P}$ i.e., generated by those local forms that satisfy the integrability condition with respect to $\mathcal{P}$. The derived system is, of course, a Pfaffian sub-system of the initially given system.

Iterating this procedure, we obtain a descending chain of Pfaffian systems terminating by an integrable system (its derived system coincides with the system) that, eventually, reduces to the null system.

We can now apply the above inquiry, concerning integrable elements, to both characteristic and derived systems though, presently, we shall only highlight the most relevant facts and, among them, those facts that were placed in evidence by Cartan (pg.9, n.6 and the following pages). We first observe, by simply inspecting the contra-variant definition, that the characteristic system is equal, at each point, to the union of those 1-integral elements (or tangent vectors) of $\mathcal{P}$ that are in involution with all the other 1-integral elements of $\mathcal{P}$.

The characteristic system is always integrable (involutive) independently of the nature of $\mathcal{P}$ and, if moreover it is regular, we can factor locally its integral foliation.

Furthermore, we can also factor all the differential forms of $\mathcal{P}$, modulo the leaves, and thereafter the initially given system factors as well to an equivalent quotient system $\tilde{\mathcal{P}}$. The integral manifolds of the initial system are the pullbacks (inverse images) of the integral manifolds in the quotient. Let us also observe that the quotient system has null characteristics.

We next give a glance at the derived system. From a contra-variant point of view, the contact element determined by $\mathcal{P}_{1}$ at the point $x_{0}$ is the linear subspace generated by all the vectors annihilated by $\mathcal{P}$ together with all 
the brackets $[\xi, \eta]_{x_{0}}$ where $\xi$ and $\eta$ are two local vector fields annihilated by $\mathcal{P}$. In the contra-variant mode, the chain of successive derived spaces or systems is increasing whereas, co-variantly, it decreases.

Obviously,

$$
\mathcal{P}_{1} \subset \mathcal{P} \subset \mathcal{C H}
$$

hence, in what concerns the corresponding pseudo-groups of local automorphisms and taking into account the functoriality of the constructions, we obtain the following two inclusions:

$$
\Gamma_{\mathcal{P}} \subset \Gamma_{\mathcal{C H}} \quad \text { and } \quad \Gamma_{\mathcal{P}} \subset \Gamma_{\mathcal{P}_{1}} .
$$

We can then proceed to extend the above second inclusion so as to obtain a composition series and, eventually, a Jordan-Hölder resolution.

Making use of the results in [16], we might then simplify the integration process of $\mathcal{P}$.

\section{The PSEUdo-Groups of LOCAL AUTOMORPHISMS}

The hypotheses and the notations being as above, we shall now have a closer look into the integration process of the Pfaffian system $\mathcal{P}$. According to Lie and Cartan, we first have to choose convenient intermediate pseudogroups, in the present case these will be associated to structures, in such a way that the second inclusion in [18, Eq. (3.1)], extends to a Jordan-Hölder resolution.

As a first step, we examine the local automorphisms of the successive derived Pfaffian systems and compare these pseudo-groups with each other as well as with the automorphisms of the initially given system $\mathcal{P}$.

Let $\mathcal{P}$ be a Pfaffian system defined on the $n$-dimensional manifold $M$ and let us assume, at least for the time being, that it is regular i.e., that $\operatorname{rank} \mathcal{P}_{x}=\operatorname{rank} \mathcal{P}_{y}$ for any two arbitrary points $x, y \in M$ where the rank is equal to the dimension of the fibre.

We then consider a local diffeomorphism (referred to as a local transformation) $\varphi: \mathcal{U} \rightarrow \mathcal{V}$, where $\mathcal{U}$ and $\mathcal{V}$ are open subsets in $M$ and the transformation $\varphi$ is assumed to be differentiable.

To say that $\varphi$ preserves or leaves invariant the Pfaffian system $\mathcal{P}$ means, of course, that $\varphi^{*}\left(\mathcal{P}_{y}\right)=\mathcal{P}_{x}$ as soon as $\varphi(x)=y$. We shall also refer to $\varphi$ as being an automorphism of $\mathcal{P}$. Observing that the linear automorphism

$$
\varphi_{x}^{*}: \mathcal{P}_{y} \rightarrow \mathcal{P}_{x}
$$


is entirely determined by the 1 -jet $j_{1} \varphi(x)$ (in fact, it is essentially the same thing), we infer that any local transformation $\varphi$, for which $j_{1} \varphi(x)$ transposes to an automorphism pertaining to $L\left(\mathcal{P}_{y}, \mathcal{P}_{x}\right)$, belongs to $\Gamma_{\mathcal{P}}$ and, consequently, this pseudo-group is a Lie pseudo-group of order $1 .^{2}$

Our next task is to show that the Lie groupoid composed by all the 1-jets of the elements belonging to $\Gamma_{\mathcal{P}}$ is an involutive first order partial differential equation in the sense of Élie Cartan, [22]. Let us denote by $\mathbf{G}_{\mathcal{P}}^{1}$ this first order groupoid associated to $\Gamma_{\mathcal{P}}$ and let us recall that the standard prolongation of the differential equation $\mathbf{G}_{\mathcal{P}}^{1}$ is the set of all second order jets $j_{2} \psi(x)$, of local maps $\psi$ of $M$, such that the Ehresmann flow

$$
y \longmapsto j_{1} \psi(y)
$$

is tangent, at first order and at the point $x$, to the equation $\mathbf{G}_{\mathcal{P}}^{1}$.

This means, in denoting by $\tilde{\psi}$ the above flow, that the image sub-manifold of $\tilde{\psi}$ is tangent to $\mathbf{G}_{\mathcal{P}}^{1}$ at the point $\tilde{\psi}(x)\left(\tilde{\psi}_{*}\left(T_{x} M\right) \subset T_{\tilde{\psi}(x)} \mathbf{G}_{\mathcal{P}}^{1}\right)$. We can define inasmuch the standard prolongations of the higher order jet groupoids associated to the pseudo-group $\Gamma_{\mathcal{P}}$ and a straightforward argument shows the following lemma.

Lemma 10.1. All the $k$-th order groupoids, $k>1$, associated to the pseudo-group $\Gamma_{\mathcal{P}}$ are the successive standard prolongations of the first order groupoid $\boldsymbol{G}_{\mathcal{P}}^{1}$

More important, we want to show that the first order groupoid as well as all the higher order groupoids are involutive equations in the sense of Cartan. The above Lemma being a first step, we now have to show that all the linear symbols of these equations are involutive i.e., that they are 2-acyclic. A rather long juggling with the techniques found in [22] including the application, to the previous equations, of the Spencer linear complex will eventually prove the following result. ${ }^{3}$

Lemma 10.2. All the groupoids associated to the Lie pseudo-group $\Gamma_{\mathcal{P}}$ are involutive.

Let us next consider the derived sequence associated to $\mathcal{P}$. It is a descending chain

$$
\mathcal{P} \supset \mathcal{P}_{1} \supset \cdots \supset \mathcal{P}_{\mu}=\mathcal{P}_{\mu+1}
$$

\footnotetext{
${ }^{2}$ The groupoid of first order jets is a differentiable manifold, the source and target maps are submersions (more precisely, surmersions) and the composition operation as well as the passage to inverses are both differentiable mappings the latter being a diffeomorphism.

${ }^{3}$ We do not provide the details of the proof since it is rather long and the result, in itself, is just auxiliary and not of first order importance.
} 
the last term being integrable or eventually null and, by the naturality of the constructions, we conclude that

$$
\Gamma_{\mathcal{P}_{\mu}} \supset \cdots \supset \Gamma_{\mathcal{P}_{1}} \supset \Gamma_{\mathcal{P}}
$$

and consequently that

$$
\mathbf{G}_{\mathcal{P}_{\mu}}^{k} \supset \cdots \supset \mathbf{G}_{\mathcal{P}_{1}}^{k} \supset \mathbf{G}_{\mathcal{P}}^{k}
$$

for all integers $k \geq 1$. In general, there is no reason for the previous sequence to be a composition series (each term being normal in the preceding one) though we shall exhibit a rather outstanding situation where not only this is true but, furthermore, the series becomes Jordan-Hölder.

Let us now assume that the Pfaffian system $\mathcal{P}$ is a flag system i.e., $\operatorname{rank} \mathcal{P}_{i}=\operatorname{rank} \mathcal{P}_{i+1}+1$ for all $i$. Then, with the notations of the reference [19], the finite and infinitesimal automorphisms pseudo-groups and pseudo-algebras of any of the factored derived systems $\overline{\mathcal{P}}_{\mu}$ (modulo their characteristic variables) are canonically equivalent (isomorphic), via a natural merihedric prolongation algorithm, to those of $\mathcal{P}$ and consequently all the corresponding higher order groupoids and algebroids are also canonically isomorphic.

However, when these derived systems are considered on their ambient space $M$, new automorphisms do appear namely, those of the associated characteristic systems that are also automorphisms of the derived systems in evidence. A characteristic system being always integrable, its automorphisms are, locally, those that leave invariant (i.e., permute) the fibres of the fibration obtained by integrating the characteristics and, still more locally ${ }^{4}$, those that leave invariant (permute) the parallel spaces to one of the components in a product.

In terms of local coordinates, we consider all the local transformations in an $n$-space, a simple pseudo-group according to Cartan, and just retain those transformations that maintain a certain restricted number of coordinates depending only upon these restricted coordinates, the resulting pseudo-group being as well simple. The quotients of these pseudo-groups and, inasmuch, the quotients of the groupoids are therefore simple and the sequence

$$
\mathbf{G}_{\mathcal{P}_{\mu}}^{k} \supset \cdots \supset \mathbf{G}_{\mathcal{P}_{1}}^{k} \supset \mathbf{G}_{\mathcal{P}}^{k}
$$

is Jordan-Hölder. The same argumentation holds on the infinitesimal level and the integration of the system $\mathcal{P}$ will entirely depend upon the nature of the quotient groupoids and their algebroids.

Let $E_{\rho_{k}}$ be a maximal integral contact element of $\mathcal{P}$ at the point $x_{0}$ and $\mathcal{I}_{\rho_{k}}$ a local integral sub-manifold of maximal dimension, tangent to

\footnotetext{
${ }^{4}$ using a cubical neighborhood
} 
$E_{\rho_{k}}$. Then, of course, $\mathcal{I}_{\rho_{k}}$ is also an integral sub-manifold of the derived system, though not forcibly maximal, and will be contained in maximal integral manifolds (not all) of this derived system. When the flag system is of maximum length i.e., when $\mathcal{P}_{\mu}=0$, then the last non-trivial system $\mathcal{P}_{\mu-1}$ is locally isomorphic to the Darboux system on $\mathbb{R}^{3}$ i.e., the system generated by the contact form $d x^{2}+x^{3} d x^{1}$. We infer that the integral elements of $\mathcal{P}$ are all 1-dimensional and that the only integral manifolds are curves namely, those curves that are tangent to the distribution $\mathcal{P}^{\perp}$.

\section{Pfaffian systems whose characters are equal to one}

We now assume that $\rho_{k}=n-r-1$, where we recall that $n=\operatorname{dim} M$ and $r=\operatorname{rank} \mathcal{P}$. In other terms, the dimension of $E_{\rho_{k}}$ and consequently also that of $\mathcal{I}_{\rho_{k}}$ are one unit less than the dimension of $\Sigma_{x_{0}}$, the contact element at the point $x_{0}$ annihilated by $\mathcal{P}_{x_{0}}$. Let us show that $\operatorname{rank} \mathcal{P}_{1}=r-1$ and provide a criterion for the integrability of this derived system. Cartan and, earlier, von Weber already gave such a criterion that Cartan claimed to be un fait très remarquable.

Firstly, since $\mathcal{P}$ is not integrable (involutive) on account of the maximality of $E_{\rho_{k}}$, it follows that $\operatorname{rank} \mathcal{P}_{1} \leq r-1$. On the other hand, since the dimension of $\mathcal{I}_{\rho_{k}}$ is equal to $n-r-1$ and all the forms $\omega^{i}$ vanish on this integral manifold, we can assume relabeling if necessary by $\left\{\varphi^{i}\right\}$ the local basis of $\mathcal{P}$, that $\mathcal{P}_{1}$ is generated by $\left\{\varphi^{2}, \cdots, \varphi^{r}\right\}$. In fact, choosing a local section $\varphi_{1}$ of $\mathcal{P}$ verifying $d \varphi_{1} \mid \Sigma_{x_{0}} \neq 0$, we can argue, together with Cartan, without performing the calculations.

Secondly and returning to the consideration of the integral contact elements, we consider at the point $x_{0}$ a maximal integral contact element $E_{\rho_{k}}$. As evidenced previously, this element is equal to the tangent space, at $x_{0}$, of an integral sub-manifold $W$ of the system $\mathcal{P}$. Locally, this sub-manifold is integral with respect to the forms $\omega^{i}$, of a local basis of $\mathcal{P}$, and some additional independent forms $\varphi^{s}$ that restrict to a single form $\varphi$ in the case when the character of the system is equal to one. But then, for the Pfaffian system $\mathcal{Q}$ generated by these forms, we can write

$$
d \omega^{i} \equiv c^{i} \mu^{i} \wedge \varphi \quad \bmod \mathcal{P},
$$

with certain functions $c^{i}$.

Consequently, the forms belonging to the sub-module of $\Gamma(\mathcal{P})$ defined by the equation $\sum c^{i} \omega^{i}=0$ are precisely those that belong to $\mathcal{P}_{1}$. This system is under certain conditions integrable namely, when its gender is larger than 1. This invariant, already introduced by von Weber, has to do with the "number" of differential 1-forms independent from those forming $\mathcal{P}$ and that enter into the expressions of $d \omega \bmod \mathcal{P}, \omega \in \Gamma \mathcal{P}$ (local sections). 
We shall consider this invariant only further but define it right below. Taking a maximal integral manifold of $\mathcal{P}_{1}$, when this system is integrable, and containing $x_{0}$, we can restrict all the data to this manifold, the investigation of the properties of $\mathcal{P}$ becoming thereafter considerably facilitated. We shall also examine further those Pfaffian systems with characters equal to two or more but presently we shall exhibit Cartan's co-variant version of the former discussion.

Definition 11.1. The gender (genre) of a local exterior differential form $\Omega$, with respect to a Pfaffian system $\mathcal{P}$, is the smallest integer $\mathbf{h}$ such that

$$
\Omega^{\mathbf{h}+1} \equiv 0 \bmod \mathcal{P}
$$

(the exponent refers to wedge products). The gender of a family of local exterior differential forms is the maximum value of the gender of its elements.

When $\mathcal{P}$ is locally generated by $\left\{\omega^{1}, \cdots, \omega^{r}\right\}$, then the above condition can be restated by the equality $\omega^{1} \wedge \cdots \wedge \omega^{r} \wedge \Omega^{\mathbf{h}+1}=0$ for the exterior forms defined on open sets contained in the domain of the generators $\omega^{i}$. The gender of a Pfaffian system $\mathcal{P}$ is equal to the gender of the family $d \omega$ where $\omega$ is an arbitrary local section of $\mathcal{P}$ and, of course, it is given by the gender of any system of generators. We also observe that the system $\mathcal{P}$ is integrable if and only if it is of gender zero ([2]).

\section{The Cartan CO-Variant approach}

We start by considering the linear 1-dimensional elements belonging to $\mathcal{P}$ namely, all the 1-dimensional sub-spaces of $\mathcal{P}$ at a given point $x_{0}$ and inquire which of them should be considered as 1-dimensional integral elements. Let $\omega \in \Gamma(\mathcal{P})$ be a generator of such a linear element i.e., $\omega_{x_{0}}$ generates this element, and let us imagine that its annihilator ker $\omega_{x_{0}}$ is an integral element for the Pfaffian system generated by $\omega$. Then the condition reads $(d \omega)_{x_{0}} \equiv 0 \bmod \omega_{x_{0}}$. It seems however more adequate, for reasons that shall be clarified hereafter, to assume the stricter requirement for the integral elements namely,

$$
(d \omega)_{x_{0}} \equiv 0 \bmod \mathcal{P} .
$$

In terms of local generators, the above condition reads

$$
(d \omega)_{x_{0}} \equiv 0 \bmod \left\{\omega_{1, x_{0}}, \cdots, \omega_{r, x_{0}}\right\} .
$$

Next, we inquire which should be considered as being the 2-dimensional integral elements containing a given (fixed) 1-dimensional element. Though entering in conflict with the notations adopted previously in the contravariant discussion, we shall keep these notations in order to conform to 
Cartan's writing. We choose a given (fixed) though arbitrary linear integral element $E_{1}$ and consider the sub-space of all those linear integral elements that are in involution with the given element. Inasmuch as in the contravariant setting, not all the linear integral elements are suitable and the condition for this to be so is, of course, the integrability condition involving the two elements.

More precisely, the co-vector $\omega_{x_{0}}, \omega \in \Gamma(\mathcal{P})^{5}$, or the sub-space generated by it, is in involution with $E_{1}$ when, by definition,

$$
\omega_{x_{0}} \equiv 0 \bmod \mathcal{P}, \quad(d \omega)_{x_{0}} \equiv 0 \bmod \mathcal{P}, \quad d \omega_{0} \equiv 0 \bmod \mathcal{P},
$$

where $\omega_{0}$ is chosen so as to induce a non vanishing co-vector belonging to $E_{1}$. In other words, this simply means that $\omega \in \Gamma(\mathcal{P})$ and that

$$
d \omega \wedge \varphi^{1} \wedge \cdots \wedge \varphi^{r}=0
$$

the same holding for $\omega_{0}$.

We can now choose $E_{2}$, containing $E_{1}$, only composed by co-vectors that are in involution with $E_{1}$ and consider the space of all those co-vectors, belonging to $\mathcal{P}$, that are simultaneously in involution with all the elements of $E_{2}$.

Continuing this process, we choose an element $E_{3}$ containing $E_{2}$ only composed by co-vectors that are in involution with all the elements of $E_{2}$ and so forth. The character $s_{1}$ (cf. Definition 7.1) defined by Cartan as being the integer satisfying $n-r-s_{1}=\operatorname{dim} \tilde{E}_{1}$, where the latter is the space of all the integral elements in involution with $E_{1}$, is then equal to $\rho_{k}$ in the contra-variant setting and both situations, the co-variant and the contra-variant, are mirror images one of the other with respect to $\mathcal{P}$.

Furthermore, the result of the previous section concerning the derived system has, in this context, a very simple proof. In the next sections we discuss Pfaffian systems with characters larger than one that provide a wide spectrum of different situations. We shall nevertheless keep within the Cartan co-variant setting since, as already mentioned earlier, the discussion as well as the calculations become straightforward and simple though we stop understanding anything since we are rowing against the tide.

From what was shown in the previous section, we can state the following

Lemma 12.1. A necessary and sufficient condition that the character of a Pfaffian system $\mathcal{P}$ be equal to one is that the rank of its derived system $\mathcal{P}_{1}$ be one unit less than its own rank.

\footnotetext{
${ }^{5}$ This form $\omega$ has nothing in common with the form $\omega$ chosen previously for the 1dimensional case.
} 


\section{Integration of Pfaffian systems With CHARACTER EQUAL to ONE}

We start by taking a local basis $\left\{\omega^{i}\right\}$ for the Pfaffian system $\mathcal{P}$ such that $\left\{\omega^{2}, \cdots, \omega^{r}\right\}$ generates its associated derived system $\mathcal{P}_{1}$, complete this basis to a local co-frame by adding the 1 -forms $\left\{\bar{\omega}^{j}\right\}$ and continue to assume that the characteristics of $\mathcal{P}$ are null.

Let us first prove the result that Cartan considered so much surprising, recalling that $\mathbf{h}$ denotes the gender of a Pfaffian system.

Lemma 13.1. A necessary and sufficient condition that the first derived system $\mathcal{P}_{1}$ of a Pfaffian system $\mathcal{P}$, of character equal to 1 , be integrable is that $\mathbf{h} \geq 2$.

Proof. Adapting the generators of $\mathcal{P}$ in such a way that the forms $\left\{\omega^{i}\right\}$, $i \geq 2$, generate $\mathcal{P}_{1}$, we have by definition

$$
d \omega^{i} \equiv 0 \bmod \left\{\omega^{1}, \cdots, \omega^{r}\right\}, \quad i \geq 2,
$$

and write, to begin,

$$
d \omega^{2} \equiv \chi \wedge \omega^{1} \bmod \left\{\omega^{2}, \cdots, \omega^{r}\right\}
$$

where $\chi$ can be taken to be a linear combination of the forms $\bar{\omega}^{j}$. A second differentiation then yields

$$
d \chi \wedge \omega^{1}+\chi \wedge d \omega^{1} \equiv 0 \bmod \left\{\omega^{2}, \cdots, \omega^{r}\right\}
$$

hence

$$
\chi \wedge d \omega^{1} \equiv 0 \bmod \left\{\omega^{1}, \cdots, \omega^{r}\right\} .
$$

We next assume that $\chi$ and $\omega^{1}$ are independent. Since $\chi$ only contain terms in $\bar{\omega}^{j}$, we can write $\chi \wedge d \omega^{1}=\mu+\eta$ where each term of $\mu$ contains some element $\omega^{i}$ and $\eta$ only contains terms that are expressed by means of the $\bar{\omega}^{j}$. Moreover, the term $\eta$ is equal to the product $\chi \wedge \bar{\eta}$, where $\bar{\eta}$ is the sum of all the terms in $d \omega^{1}$ that are written only with the help of the forms $\bar{\omega}^{j}$. It then follows that the exterior product $d \omega^{1} \wedge d \omega^{1}$ presents, in its unique term not containing any form $\omega^{i}$, a double product $\chi \wedge \chi$ hence this term vanishes and the double product above becomes equal to:

$$
d \omega^{1} \wedge d \omega^{1} \equiv 0 \bmod \left\{\omega^{1}, \cdots, \omega^{r}\right\} .
$$

Consequently the gender of $\mathcal{P}$ is at most equal to 1 contradicting the initial hypothesis. We infer that $\chi$ and $\omega^{1}$ are dependent and that the relation [18, Eq. (6.2)] reduces to

$$
d \omega^{2} \equiv 0 \bmod \left\{\omega^{2}, \cdots, \omega^{r}\right\} .
$$

The same argument being valid for all the other forms generating $\mathcal{P}_{1}$, the integrability of this derived system then follows. 
We can now proceed with the integration of the system $\mathcal{P}$ namely, construct integral manifolds of dimension $n-r-1$ that are tangent to given maximal integral contact elements, also of the same dimension, since the character of $\mathcal{P}$ is assumed to be equal to one.

Fixing the point $x_{0}$, we denote by $\mathcal{L}$ the integral leaf of $\mathcal{P}_{1}$ that contains this given point. Then $\operatorname{dim} \mathcal{L}=n-r+1$ and, if we assume as previously that the forms $\omega^{i}, i \geq 2$, generate $\mathcal{P}_{1}$, the restricted system $\mathcal{P} \mid \mathcal{L}$ is generated by the single 1 -form $\omega^{1} \mid \mathcal{L}$.

Obviously, any maximal integral element issued at the point $x_{0}$ is contained in $T_{x_{0}} \mathcal{L}$ and any maximal integral manifold of $\mathcal{P}$ containing $x_{0}$ is contained in $\mathcal{L}$ being as well an integral manifold of the restricted system.

Moreover, the maximality properties are preserved under restriction since the restricted system is not integrable, otherwise there would be an $(n-r)$ dimensional integral manifold of $\mathcal{P}$ passing through $x_{0}$. Since the gender of a Pfaffian system is a point-wise notion involving only the exterior algebra of the co-tangent spaces at given points, we infer that the gender of the restricted system is the same as that of the initial system.

On the other hand, the restricted system $\mathcal{P} \mid \mathcal{L}$ is non-integrable and has rank equal to one hence is a Darboux system, [19]. The definition, per se, of the gender of this restricted system will then assert that $\mathcal{P} \mid \mathcal{L}$ is a Darboux system of Cartan or, inasmuch, a system of Darboux class equal to $2 \mathbf{h}+1$ hence generated locally by a form

$$
d z^{h+1}+p^{1} d z^{1}+p^{2} d z^{2}+\cdots+p^{h} d z^{h}
$$

where $h=\mathbf{h}$ is the gender at a given point ${ }^{6}$. Putting all the stacks together, we infer that $\mathcal{P}$ admits local models of the form:

$$
\begin{aligned}
& d y^{1} \\
& d y^{2} \\
& \cdots \\
& d y^{r-1} \\
& d z^{\mathbf{h}+1}+p^{1} d z^{1}+p^{2} d z^{2}+\cdots+p^{h} d z^{h}
\end{aligned}
$$

with respect to suitable local coordinates and where the functions

$$
y^{1}, y^{2}, \cdots, y^{r-1}
$$

are chosen to be independent first integrals of the (integrable) derived system, $c f$. [2, pg.28]. The solutions of the above system will provide, locally, the maximal integral manifolds of $\mathcal{P}$. If by any chance the given Pfaffian

\footnotetext{
${ }^{6}$ Cartan preferred minus signs.
} 
system admits non-vanishing characteristics ${ }^{7}$, then it suffices to chose, for $y, z$ and $p$, first integrals of the characteristic system.

Let us finally examine what happens when $\mathbf{h}=1$. We can in this case integrate, starting from a given point $x_{0}$ on $\mathcal{L}$, all the vector fields belonging to the annihilator of $\mathcal{P} \mid \mathcal{L}$. Denoting by

$$
t \longmapsto\left(z^{1}(t), \cdots, z^{\mu}(t)\right), \quad \mu=n-r+1,
$$

a generic integral curve commencing at $x_{0}$, we plot all the points obtained by considering the images of the above integral curves conditioned to a given specific, though arbitrary, non-trivial relation among the targets $z^{i}$. A detailed proof of this statement is rather long and shall be omitted. Fortunately, the author is backed up by Cartan's fantastic intuition, see [2, pg.28-29], where he refers to von Weber [31].

\section{Pfaffian systems Whose characters are larger than one}

We begin by examining systems with characters equal to 2 and, to simplify, we assume right away that their characteristics are null. Much will then depend upon the gender of the system and offers several options. We exhibit firstly conditions under which the character being equal to $2 \mathrm{im}$ plies that the rank of the first derived system is equal to $r-2$, the general rule not being as strict in the present case as it was previously. It seems furthermore worthwhile, at this point, to make a few comments since our statement is, apparently, in disagreement with Cartan's claims.

Let us assume, for a moment, that the Pfaffian system $\mathcal{P}$ has non-trivial characteristics and let us denote by $\mu$ the difference in the dimensions of the initially given manifold $M$ and the (local) quotient manifold $\bar{M}$, modulo the characteristics of the system $\mathcal{P}$. Then $\mathcal{P}$ factors to an equivalent system $\overline{\mathcal{P}}$, both systems have the same rank and, consequently, the annihilator distributions $\Sigma$ and $\bar{\Sigma}$ differ, in their point-wise dimensions, by $\mu$, the dimension of $\bar{\Sigma}$ being lesser since the characteristic distribution is contained in $\Sigma$.

Similar statements hold for the derived system and its quotient in $\bar{M}$ though the latter can still have non-trivial characteristics thus explaining the discrepancy concerning the rank of $\overline{\mathcal{P}_{1}}$ and the dimensions of the maximal integral elements of $\mathcal{P}$ and $\overline{\mathcal{P}}$. We also observe that the passage to the derived systems is functorial and therefore compatible with quotients i.e., $\overline{\mathcal{P}_{1}}=(\overline{\mathcal{P}})_{1}$.

In the sequel we shall only give a brief account on Cartan's results so as to promptly continue with our main discussion. In what concerns the Pfaffian systems of character 2, the main result is the following:

\footnotetext{
${ }^{7}$ Cartan never imposed any restriction on the characteristics since, in his modus operandi, nothing much would change.
} 
Theorem 14.1 (Cartan). The rank of the derived system of a Pfaffian system $\mathcal{P}$ of character two is always two units less than the rank of the system except when $\mathcal{P}$ admits characteristic elements with dimensions not less than $\operatorname{dim} \Sigma_{x_{0}}-3$ in which case the rank lowers by at least three units.

The proof is similar to that of the previous lemma as soon as we choose two independent local sections of the system whose differentials do not vanish on the kernel and an entirely analogous result also holds in general. We shall therefore only give some attention to the second part of the statement that is far more delicate.

Let us then assume, the Pfaffian system being of character 2, that the rank of the derived system $\mathcal{P}_{1}$ is equal to $r-\mu$ where $\mu \geq 3$. This being so, we can choose a local basis $\left\{\omega^{1}, \cdots, \omega^{\mu}, \cdots, \omega^{r}\right\}$ for $\mathcal{P}$, in a neighborhood of the point $x_{0}$, in such a way that the forms $\left\{\omega^{1}, \cdots, \omega^{\mu}\right\}$ do not belong to $\mathcal{P}_{1}$, hence inasmuch any non-trivial linear combination of these forms cannot belong to the derived system, the remaining forms generating $\mathcal{P}_{1}$. Since $d \omega^{j} \not \equiv 0 \bmod \mathcal{P}, j \leq \mu$, we can select $\mu$ independent vectors $v_{j}$ in $\Sigma_{x_{0}}$ not belonging, of course, to the characteristic sub-space of $\mathcal{P}$ at the point $x_{0}$ and in such a way that the family

$$
\left\{\omega^{1}, \cdots, \omega^{r}, i\left(v_{1}\right) d \omega^{1}, \cdots, i\left(v_{\mu}\right) d \omega^{\mu}\right\}
$$

is a local basis for the characteristic system of $\mathcal{P}$. The rank $r+\mu$ of this characteristic system must be equal to $n$, hence $\mu=n-r$ otherwise the system $\mathcal{P}$ would have non-trivial characteristics contradicting our assumption. But then, the system $\mathcal{P}$ will have rank equal to the dimension of $M$, its annihilator is the null distribution and the integral leaves are the points of $M$, a rather uninteresting foliation. Within the scope of our assumptions, we can therefore restate the above theorem as follows:

Theorem 14.2. Let $\mathcal{P}$ be a Pfaffian system with null characteristics and character equal to 2 . Then the rank of its derived system is two units less than the rank of the system.

Let us now say a word on the number 3. Apart from being an extraordinary prime number that brings much luck, let us argue as follows.

We select, among all the possible non-trivial linear combinations of the forms $\left\{\omega^{1}, \cdots, \omega^{\mu}\right\}$, one such form that has a minimum gender at the point $x_{0}$. Re-arranging once more the local basis, we can assume for convenience that the above form is simply $\omega^{1}$ and can therefore write, at the point $x_{0}$, $\left(d \omega^{1}\right)^{\mathbf{h}+1} \equiv 0 \bmod \left\{\omega^{1}, \cdots, \omega^{r}\right\}, \mathbf{h}$ being the minimum value for which the above congruence holds.

A simple calculation will then show that the value $\mathbf{h}=1$ is the only possible value for which the rank of the characteristic system is larger than 
$r-2$ and consequently the rank of the derived system is lesser than $r-2$, hence precisely equal to $r-3$.

However, we should mention that, in many applications, Pfaffian systems with non-trivial characteristics do appear most naturally and, furthermore, the factoring of a Pfaffian system, modulo its characteristics, is never an easy task since it requires an integration process that only reduces locally to a system of ordinary differential equations when the characteristic leaves are 1-dimensional.

Cartan also discusses those Pfaffian systems for which half of the pointwise dimension of their annihilators $\Sigma$ is not less than the value of their characters. Since, once more, the discussion of this setup involves nonvanishing characteristics, we shall leave it aside. There is however a special case when the equality of the above mentioned values holds and that merits to be commented. Let us then assume that the previously mentioned system $\mathcal{P}$ also verifies the following properties:

(a) The character of $\mathcal{P}$ is equal to 2 ,

(b) $\mathcal{P}$ has null characteristics and

(c) All the maximal integral contact elements that contain a specific 1-dimensional integral element $E_{1}$ also contain an integral element $E_{2} \supset E_{1}$ whose dimension is not less than 2.

Under these conditions, the equality $\frac{n-r}{2}=\rho_{k}$ holds. Translated in terms of maximal integral manifolds, the condition (c) means that if two such manifolds have in common a curve they also have in common at least a surface. Cartan calls such systems systatical (systatiques).

\section{Singular Pfaffian systems}

For generic Pfaffian systems, the successive characters assume their maximum values hence, in particular, for systems with character equal to 2 the values are:

$$
s_{1}=s_{2}=\cdots=s_{\rho_{k}-1}=2 .
$$

As for $s_{\rho_{k}}$, it is equal to zero, one or two according to the remainder of the division of $n-r$ by 3 . This being so, $s_{n}=2$ when $n-r=3 \rho_{k}+2, s_{n}=1$ when $n-r=3 \rho_{k}+1$, and $s_{n}=0$ when $n-r=3 \rho_{k}$.

We shall say, together with Cartan, that the Pfaffian system $\mathcal{P}$, with character equal to 2 , is singular when $s_{\rho_{k}-1} \leq 1$. Still quoting Cartan, for a singular system having character two and null characteristics the maximum dimension of the linear integral contact elements i.e., the value of $\rho_{k}$ is at least equal to 3 and, consequently, the point-wise dimension of the distribution $\Sigma$ is at least equal to 6 . Another rather surprising property, consequence of the above assertions, is the following: 
Proposition 15.1. All the systatical Pfaffian systems are singular as soon as $\rho_{k} \geq 3$.

Cartan's Mémoire then terminates with an attempt to extend some of the above properties to non-systatical systems and culminates in the following statement:

A singular Pfaffian system with null Cauchy characteristics and character equal to 2 can be integrated by means of a system of ordinary differential equations. In case the system admits non-trivial characteristics, its characteristic system can also be integrated by means of ordinary differential equations whenever the derived system of the given system is non-integrable.

Curiously enough, the second order Monge characteristics play an important role in the proofs.

We finally arrive at what really matters namely, the integration of the above considered Pfaffian systems via the reductions provided by JordanHölder resolutions ([14]). In the previous discussion, we have seen to what extent the derived systems are relevant. Consequently, we begin by integrating the derived system and afterwards locate, among the manifolds thus obtained, those that are maximal for the given system. We are thus faced in constructing Jordan-Hölder resolutions for the successive derived systems and each specific situation will exhibit its particular techniques. It might be however of some advantage to try initially integrating one of the systems that makes part of the sequence of iterated derived systems and, in this case, the chain [18, Eq. (10.3)], will already provide some useful terms. In particular, it is possible to detect the appearance of ordinary differential equations, these showing up when the quotients are 1-dimensional.

\section{EXAMPLES}

1. Allowing initially $\operatorname{dim} M=5$ and $\operatorname{rank} \mathcal{P}=3$, the system generated by

$$
\left\{\omega^{1}=d x^{1}+x^{4} d x^{5}, \quad \omega^{2}=d x^{2}, \quad \omega^{3}=d x^{3}\right\}
$$

is not integrable since

$$
d \omega^{1}=d x^{4} \wedge d x^{5} \not \equiv 0 \bmod \left\{\omega^{1}, \omega^{2}, \omega^{3}\right\}
$$

and $\mathcal{P}_{1}$ is generated by $\left\{\omega^{2}, \omega^{3}\right\}$ hence is, of course, integrable. The character of $\mathcal{P}$ is equal to 1 since, at any point $x_{0}$, the maximal integral manifold is the line defined by

$$
x^{1}=c^{1}, \quad x^{2}=c^{2}, \quad x^{3}=c^{3}, \quad x^{5}=c^{5}
$$

for some constants $c^{i}$ and is a line parallel to the $x^{4}$-axis. It should be observed that the annihilator $\Sigma_{x_{0}}$ of $\mathcal{P}_{x_{0}}$ is 2-dimensional. 
2. Let us now replace $\omega^{3}$ by $\tilde{\omega}^{3}=d x^{3}+x^{5} d x^{1}$. Then

$$
d \tilde{\omega}^{3}=d x^{5} \wedge d x^{1}=d x^{5} \wedge \omega^{1},
$$

hence $\mathcal{P}_{1}$ is again generated by $\left\{\omega^{2}, \omega^{3}\right\}$ but is not integrable since

$$
d \tilde{\omega}^{3} \not \equiv 0 \bmod \left\{\omega^{2}, \omega^{3}\right\} .
$$

This derived system, whose annihilator is point-wise 3-dimensional, just admits the 2-dimensional integral manifolds defined by the equations

$$
x^{1}=c^{1}, \quad x^{2}=c^{2}, \quad x^{3}=c^{3} .
$$

3. We next give a glance at those Pfaffian systems with character equal to 2 and it will just suffice to add one more dimension to our space. Let us therefore consider the system $\mathcal{P}$ generated, on a 6 -space, by

$$
\left\{\omega^{1}=d x^{1}+x^{4} d x^{5}, \quad \omega^{2}=d x^{2}+x^{5} d x^{6}, \quad \omega^{3}=d x^{3}\right\}
$$

Then the annihilator $\Sigma_{x_{0}}$, of $\mathcal{P}$, is 3-dimensional, the derived system $\mathcal{P}_{1}$ is generated by $\left\{d x^{3}\right\}$ hence is integrable and its rank is two units less than the rank of $\mathcal{P}$. Consequently, $\mathcal{P}$ is not integrable. On the other hand, the generators vanish on the affine line defined by the equations

$$
x^{1}=c^{1}, \quad x^{2}=c^{2}, \quad x^{3}=c^{3}, \quad x^{5}=c^{5}, \quad x^{6}=c^{6}
$$

and, moreover, this line is a maximal integral manifold. The character of $\mathcal{P}$ is therefore equal to 2 .

4. Let us now replace $\omega^{3}$ by $\tilde{\omega}^{3}=d x^{3}+x^{5} d x^{1}$. Then $\mathcal{P}$ continues to be of character 2 but the derived system $\mathcal{P}_{1}$, generated by $\tilde{\omega}^{3}$, is no longer integrable since

$$
d \tilde{\omega}^{3}=d x^{5} \wedge d x^{1} \not \equiv 0 \bmod \left\{\tilde{\omega}^{3}\right\}
$$

and only admits the 4-dimensional maximal integral manifolds (being 4dimensional affine spaces) defined by the equations

$$
x^{1}=c^{1}, \quad x^{3}=c^{3} .
$$

The maximal integral elements of $\mathcal{P}$ are only 1-dimensional and the maximal integral manifolds are affine lines.

5. We continue our little game by constructing now a Pfaffian system of character 2 whose derived system has rank three units less than the rank of the given system i.e., we shall construct a singular Pfaffian system with character equal to 2 . For this, we simply consider the system $\mathcal{P}$ generated, on a 6 -dimensional space, by

$$
\left\{\omega^{1}=d x^{1}+x^{4} d x^{5}, \quad \omega^{2}=d x^{2}+x^{5} d x^{6}, \quad \omega^{3}=d x^{3}+x^{6} d x^{4}\right\} .
$$


The maximal integral manifolds of $\mathcal{P}$ are affine lines and a direct calculation shows that $\mathcal{P}_{1}=0$. The rank of the derived system is three units less than its own rank.

As for the gender of a Pfaffian system, the first example considered above illustrates the content of the [18, Lemma 6.2], since the local section $\omega^{1}+x^{2} \omega^{3}$ has its gender equal to 2 .

6. Let us finally terminate our discussion by exhibiting three Jordan-Hölder resolutions.

To begin with, we continue to consider the first example in 5 -space and observe that the pseudo-group $\Gamma$ of all the local automorphisms of $\mathcal{P}$ leaves also invariant the derived system $\mathcal{P}_{1}$ hence, inasmuch, its annihilator $\Sigma_{1}$ whose point-wise dimension is equal to 3 . Needless to say that the integral manifolds of $\Sigma_{1}$ are all parallel affine three dimensional sub-spaces in 5 -space. Moreover, $\Gamma$ also leaves invariant the annihilator $\Sigma \subset \Sigma_{1}$. Denote by $\Gamma_{1}$ the sub-pseudogroup of all the elements $\varphi$, belonging to $\Gamma$, that maintain invariant each connected integral manifold of $\Sigma_{1}$ i.e., $\varphi$ transforms the points of such an integral manifold into points of the same manifold. Equivalently, this simply means that the above mentioned integral manifolds are the intransitivity classes of $\Gamma_{1}$.

We can now factor, locally, the given 5 -space modulo these intransitivity classes and obtain, as quotient, an open set $U$ in numerical 2-space. Furthermore, the Pfaffian system $\mathcal{P}$ being invariant under $\Gamma_{1}$, factors to the quotient and yields a quotient Pfaffian system $\bar{P}$ that is generated by $\left\{d x^{1}, d x^{2}\right\}$ hence its maximal integral leaves are the points of $U$. Fixing a point $y_{0} \in U$ and denoting by $Y_{0}$ the inverse image $q^{-1}\left(y_{0}\right)$, where $q$ is the quotient projection modulo the above intransitivity classes, we can now restrict the system $\mathcal{P}$ to the sub-manifold $Y_{0}$, this restricted system $\mathcal{Q}$ being generated by the 1 -form $d x^{1}+x^{4} d x^{5}$. Since $\operatorname{dim} Y_{0}=3$ and since the generating form of the system is of maximum Darboux class equal to 3 , the restricted system is a Darboux system of rank 1 and only admits 1-dimensional integral manifolds that, in fact, are the affine lines in 5-space that constitute the maximal integral manifolds of $\mathcal{P}$.

The integration of this system is consequently achieved via the JordanHölder method since the quotient pseudo-group $\Gamma / \Gamma_{1}$ is equal to the set of all the local transformations in $U$, hence is simple.

7. We shall continue with the same Pfaffian system but apply the JordanHölder methodology in a different manner. The character of the system being equal to 1 and, consequently, the maximal integral manifolds being 1-dimensional curves that actually constitute a foliation, we consider the sub-pseudogroup $\Gamma_{1}$ as being composed by all those local transformations 
that keep invariant these connected maximal integral curves, in much the same way as those considered previously for the integrals of the first derived system.

As before, let $U$ be a local quotient of the 5-space modulo the above integral curves. Then the dimension of $U$ is equal to 4 and we can, inasmuch, factor locally the system $\mathcal{P}$ to the space $U$. Since the annihilator, at each point, of the system $\mathcal{P}$ contains the tangent space to the integral curve at that point, we infer that the quotient system $\mathcal{Q}$ is still of rank 3 and is generated by $\left\{d x^{2}, d x^{3}\right\}$ together with the quotient of $\omega^{1}$. However, since the dimension of $U$ is equal to 4 and the quotient system $\mathcal{Q}$ has rank equal to 3 , we infer that it is necessarily integrable, its maximal integrals being again curves.

Proceeding as prescribed in the Jordan-Hölder scheme, we fix a certain, though arbitrary, integral curve of $\mathcal{Q}$ and consider the inverse image of this curve (more precisely, of the image of this curve) modulo the established quotient projection described above. In other terms, we consider the surface $Y$ obtained as the union of all the integral lines of $\mathcal{P}$ that project onto points of the selected (though fixed) integral curve of $\mathcal{Q}$. Knowing, a priori, that the integral curves are parallel affine lines, we shall thus obtain a ruled surface.

Restrict now $\mathcal{P}$ to the above surface and denote this restricted Pfaffian system by $\overline{\mathcal{P}}$. Since it must vanish on the tangent spaces to the integral curves, its rank must be equal to 1 and its integral curves are precisely the integral curves of $\mathcal{P}$ contained in the above surface. The Jordan-Hölder method is therefore a most outstanding integration procedure since it enables us to obtain the maximal integral manifolds of the initially given system by a very precise and constructive technique. It should be noted, inasmuch, that the whole process can of course be performed without knowing, a priori, the nature of the maximal integral manifolds that we are looking for (curves in our present context), this being most often the case and, in fact, the sole purpose of an integration method.

Moreover, for the system discussed here, the usual quadrature technique (Calculus I), will enable us to find a first integral for the integral curves.

8. Let us now consider the last example given above, where the three forms $\omega^{i}$, generating the system, are of Darboux class equal to 3 . We proceed as above and consider the sub-pseudogroup $\Gamma_{1}$ composed by all those elements of $\Gamma$ that preserve the maximal integral manifolds of $\mathcal{P}$, here again 1-dimensional curves since the character of $\mathcal{P}$ is equal to 2 .

The local quotient space $U$ is, in this case, 5-dimensional and the resulting quotient system $\mathcal{Q}$ continues to be of rank 3 , the point-wise dimension 
of its annihilator being thereafter equal to 2. However, this quotient system acquires a character equal to one ${ }^{8}$.

Taking a 1-dimensional integral curve $\gamma$ of $\mathcal{Q}$, considering the inverse image $Y=q^{-1}(\mathrm{im} \gamma)$ defined in the initially given 6 -space and restricting $\mathcal{P}$ to the surface $Y$, we finally obtain a rank 1 Pfaffian system whose integral curves are precisely the integral curves of $\mathcal{P}$ contained in $Y$ and for which a first integral can be obtained by a quadrature.

We should at present confess to the reader that the above discussion is actually a big fake. In fact, the aim of the Jordan-Hölder procedure is, of course, the obtainment of maximal integral manifolds of the given system whereas our procedure was to exhibit this technique, on examples, with the help of the a priori knowledge of the solutions. Nevertheless, we believe that the reader is able to devise much more sophisticated examples where the true aim will be, of course, the determination of maximal integral manifolds not known in advance.

One last word is due. Élie Cartan never claimed that the Jordan-Hölder procedure was an easy matter. It can in fact become extremely involved, reason for which Cartan never mentioned neither Jordan nor Hölder.

9. A very interesting type of non-integrable Pfaffian systems is given by the Flag Systems considered in various occasions by Élie Cartan, e.g., [2], [4], [5]. We claim that a flag system $\mathcal{F}$ with null characteristics has always its character equal to $n-r-1$, where $n=\operatorname{dim} M$ and $r=\operatorname{rank} \mathcal{F}$. In fact, proceeding as indicated in [19] and replacing the descending chain of derived systems by the more appropriate structured multi-fibration where each successive derived system is replaced by an equivalent system that now has also null characteristics, we see that the maximal dimensional integral manifolds, at each stage, are the integral curves contained in the inverse image of the integral curves of the Darboux system, these last curves being also the maximal dimensional integral curves.

Our claim then follows since the point-wise dimension of the annihilator distribution $\mathcal{F}^{\perp}$ is precisely $n-r$.

More generally, we can consider the Multi-Flag Systems or, still better, the Truncated Multi-Flag Systems that have much relevance in the study of under-determined ordinary differential equations in what concerns the Monge property, [20]. We leave the details to the reader and, in particular, the calculation of the character for such systems. It should be noted that the difference between flag and multi-flag systems can be recognized by looking at their local equivalence pseudo-groups that are non-isomorphic. Whereas for flag systems the Monge condition is a necessary and sufficient

\footnotetext{
${ }^{8}$ somehow, the reduction in the dimension squeezes the system into a smaller ambient space.
} 
condition for the validity of the Monge property, [5], for multi-flag systems the condition is only sufficient, [20].

10. Last but not least, let us give a glance at a rare jewel left to us by Élie Cartan namely, his article on Galois theory, [7], where he bases his argumentation on the classical Picard-Vessiot theory for linear differential systems and shows how to integrate such systems via the Jordan-Hölder method.

On the base manifold $M$, we consider the $k$-th order jet space $J_{k} T M$ of all the $k$-jets of local sections of the tangent bundle $\pi: T M \rightarrow M$, i.e., $k$-jets of local vector fields on $M$. Then $\alpha_{k}: J_{k} T M \rightarrow M, \alpha_{k}$ denoting the source map, becomes again a vector bundle, its linear operations being simply the extensions, to $k$-jets, of the linear operations in $T M$.

A linear partial differential equation of order $k$ is, by definition, a vector sub-bundle $\mathcal{R}$ of $J_{k} T M$. The dual (co-variant) version reads as follows. We take the dual bundle $T^{*} M$, consider the jet space $J_{k} T^{*} M$ and then put in evidence a vector sub-bundle $\mathcal{S}$. It should be remarked that

$$
J_{k} T^{*} M \equiv\left(J_{k} T M\right)^{*} .
$$

Given a linear differential equation $\mathcal{R} \subset J_{k} T M$, its annihilator becomes a vector sub-bundle of $J_{k} T^{*} M$ i.e., $\mathcal{R}^{\perp}=\mathcal{S} \subset J_{k} T^{*} M$ and, inasmuch, we can consider the annihilator, in $J_{k} T M$, of any sub-bundle $\mathcal{S} \subset J_{k} T^{*} M$.

Let us denote $n=\operatorname{dim} M$ and $n_{k}=\operatorname{dim} J_{k} T M$. On account of the previous remarks, it also follows that $n_{k}=\operatorname{dim} J_{k} T^{*} M$. Taking a local coordinate system $\left(U,\left(x^{i}\right)\right)$ in the base space $M$, we derive corresponding coordinate systems for $J_{k} T M$ and $J_{k} T^{*} M$, defined on the respective inverse images of the open set $U$, and any linear partial differential equation will be determined, locally, by a system of linear equations with eventually variable coefficients. If rank $\mathcal{R}=\rho$ denotes the point-wise dimension of the fibres, then $\operatorname{rank} \mathcal{S}=n_{k}-\rho$ and the number of independent linear equations defining locally the system is also equal to $n_{k}-\rho$.

As is usual, we indicate by $\mathcal{C}_{k}$ the canonical contact system defined on $J_{k} T M$ and, just to mark the distinction, by $\mathcal{C}_{k}^{*}$ the canonical contact system defined on $J_{k} T^{*} M$. We recall that a local section in the $k$-jets is holonomic i.e., results from a section in the base tangent or co-tangent bundles if and only if it is annihilated by the contact Pfaffian syatem and, furthermore, a local transformation in the jet space is the prolongation of a base space transformation (in $T M$ or $T^{*} M$ ) if and only if it preserves the corresponding contact structure.

In the present case, we shall restrict our attention to the pseudo-group of all the local transformations that are vector bundle morphisms. This is the starting point of the Picard-Vessiot theory and the set of all the 
(global) vector bundle automorphisms of the equation is called its Galois group. Most often, the localized pseudo-group operates non-transitively on the equation though the $(k+1)$-st order groupoid of all the $(k+1)$-jets that preserve the equation, via the semi-holonomic action, is always transitive on account of linearity.

This action is defined as follows. Given a jet $j_{k+1} \varphi(v)$ on $T M$ or $T^{*} M$, we consider the flow $j_{k} \varphi: w \mapsto j_{k} \varphi(w)$ and subsequently take the jet $j_{1}\left(j_{k} \varphi\right)(X), \alpha_{k}(X)=v$. Restricting the contact structures to the corresponding equations, we obtain the Pfaffian systems $\overline{\mathcal{C}}_{k}$ and $\overline{\mathcal{C}}_{k}^{*}$ associated to these given linear equations. These systems are not integrable but their $n$ th dimensional integral manifolds transversal to the $\alpha_{k}$-fibres are precidely the images of the $k$-jets of solutions.

On account of the transitivity at the groupoid level, we can apply the Jordan-Hölder integration method as outlined in [16] and [14]. When the linear pseudo-group operates transitively on the equations, the integration process can be achieved by just calling out the simple linear Lie groups. We finally observe that Picard [28], Vessiot [29], [30], and also Drach [9] only considered first order linear systems. Many years earlier, Sophus Lie studied linear differential equations invariant under linear groups with constant coefficients, [25]. As for Cartan's Mémoire, he shows us much, in [7], via examples.

\section{REFERENCES}

[1] R. Almeida, P. Molino. Suites d'Atiyah et feuilletages transversalement complets. C.R. Acad. Sci. Paris, 300:13-15, 1985 (in fre).

[2] E. Cartan. Sur l'intégration de certains systèmes de Pfaff de caractère deux. Bull. Soc. Math. de France, 29:233-302, 1901 (in fre).

[3] E. Cartan. Sur l'intégration des systèmes d'équations aux différentielles totales. Annales Sci. Ecole Norm. Sup., 18:241-311, 1901 (in fre).

[4] E. Cartan. Les systèmes de Pfaff à cinq variables et les équations aux dérivées partielles du second ordre. Annales Sci. École Norm. Sup., 27:109-192, 1910 (in fre).

[5] E. Cartan. Sur les systèmes en involution d'équations aux dérivées partielles du second ordre à une fonction inconnue de trois variables indépendantes. Bull. Soc. Math. France, 39:352-443, 1911 (in fre).

[6] E. Cartan. Sur l'équivalence absolue de certains systèmes d'équations différentielles et sur certaines familles de courbes. Bull. Soc. Math. de France, 42:12-48, 1914 (in fre).

[7] E. Cartan. La théorie de Galois et ses générlisations. Comm. Math. Helv., 11:9-25, 1938 (in fre).

[8] E. Cartan. Les systèmes différentiels extérieurs et leurs applications géométrique. Hermann, Paris, 1945 (in fre).

[9] J. Drach. Essai sur une théorie générale de l'intégration et sur la classification des transcendantes. Ann. Ecole. Norm. Sup., 15:243-384, 1898 (in fre).

[10] Ch. Ehresmann. Gattungen von lokalen Strukturen. Jahresbericht der Deutschen Mathematiker-Vereinigung, 60:49-77, 1958 (in ger). 
[11] Ch. Ehresmann. Esquisses d'un folklore de géométrie différentielle. Cahiers Topol. Géom. Différ., C. Ehresmann, 1967 (in fre).

[12] Ch. Ehresmann. Sur les catégories différentiables. Atti. Conv. Int. Geom. Diff., Bologna, 1967 (in fre).

[13] A. Kumpera. Intégration des distributions singulières sur les variétés banachiques. Istit. Analisi Globale e Applic., Consiglio Nazionale delle Ricerche, 1:1-67, 1982 (in fre).

[14] A. Kumpera. On the Lie and Cartan theory of invariant differential systems. J. Math. Sci. Univ. Tokyo, 6:229-314, 1999 (in eng).

[15] A. Kumpera. An introduction to Lie groupoids. arXiv, 1512.01454:44, 2015 (in eng).

[16] A. Kumpera. Non-integrable Pfaffian systems. arXiv, 1608.02871:25, 2015 (in eng).

[17] A. Kumpera. On The Equivalence Problem for Geometric Structures, I, II. arXiv, 1412.8391 and 8394:55+38, 2015 (in eng).

[18] A. Kumpera. On the integrability problem for systems of partial differential equations in one unknown function, i. Proceedings of the International Geometry Center, 11(4):35-71, 2018 (in eng).

[19] A. Kumpera. Automorphisms of Flag Systems. J. Differ. Eq., 266(2-3), 2019 (in eng).

[20] A. Kumpera, J. Rubin. Multi-Flag Systems and Ordinary Differential Equations. Nagoya Math. J., 166:1-27, 2002 (in eng).

[21] A. Kumpera, C. Ruiz. Sur l'équivalence locale des systèmes de Pfaff en drapeau. $F$. Gherardelli (Ed.), Monge-Ampère Equations and Related Topics, Firenze 1980, Proceedings, Istit. Naz. Alta Mat. "Francesco Severi", Roma, pages 201-248, 1982 (in fre).

[22] A. Kumpera, D. Spencer. Lie Equations, Vol.1: General Theory. Princeton University Press, 1972 (in eng).

[23] R. Kumpera. Sur la géométrie de contact d'ordre supérieur. Portugal. Math., 44(2):199 $212,1987$.

[24] S. Lie. Begründung einer Invariantentheorie der Berührungstransformationen. Math. Ann., 8:215-288, 1874 (in ger).

[25] S. Lie. Allgemeine Theorie der partiellen Differentialgleichungen erster Ordnung. Math. Ann., 9:245-296, 1875 (in ger).

[26] S. Lie. Begründung einer Invariantentheorie der Berührungstransformationen. Math. Ann., 9:289-303, 1875 (in ger).

[27] S. Lie. Klassifikation und Integration von gewönlichen Differentialgleichungen zwischen x,y, die eine Gruppe von Transformationen gestatten. Math. Ann., 32:213-281, 1888 (in ger).

[28] E. Picard. Traité d'analyse. Gauthier-Villars, Paris, 1896 (in fre).

[29] E. Vessiot. Sur la théorie de Galois et ses diverses généralisations. Ann. Ecole. Norm. Sup., 21:9-85, 1904 (in fre).

[30] E. Vessiot. Sur la réductibilité et l'intégration des systèmes complets. Ann. Ecole. Norm. Sup., 29:209-278, 1912 (in fre).

[31] E. von Weber. Zur Invariantentheorie der Systeme Pfaff'scher Gleichungen. Leipz. Ber., 50:207-229, 1898 (in ger).

Received: October 5, 2018, accepted: February 3, 2019.

Antonio Kumpera

State University of Campinas

CAmpinas, SP, BRAZIL

Email: antoniokumpera@gmail.com

ORCID: orcid.org/0000-0001-8290-4254 